\title{
1 Sociality enhances survival in science, especially for female researchers
}

2

3 Jessica E.M. van der Wal ${ }^{1,2,3^{*}}$, Rose Thorogood ${ }^{1,2,4}$ and Nicholas P.C. Horrocks ${ }^{4}$

$5 \quad{ }^{1}$ Helsinki Institute of Life Science, University of Helsinki, Helsinki, Finland

$6 \quad{ }^{2}$ Research programme in Organismal and Evolutionary Biology, Faculty of Biological and

7 Environmental Sciences, University of Helsinki, Helsinki, Finland

$8 \quad{ }^{3}$ FitzPatrick Institute of African Ornithology, University of Cape Town, South Africa

$9{ }^{4}$ Department of Zoology, University of Cambridge, Cambridge, UK

10 *e-mail: jessicavanderwal1@gmail.com

11

\section{ORCIDs:}

13 J.E.M.vdW: https://orcid.org/0000-0002-6441-3598

14 R.T: https://orcid.org/0000-0001-5010-2177

15 N.P.C.H: https://orcid.org/0000-0003-0762-4142

16

17 The authors declare that no competing interests exist. 


\section{ABSTRACT}

19 Intense competition for limited opportunities means the career path of a scientist is a

20 challenging one, and female scientists in particular are less likely to survive in academia.

21 Collaboration is a key factor in scientific advances, and in social species enhanced sociality

22 improves fitness and longevity. Yet whether sociality influences career progression and

23 survival in science, and how this might differ between genders, is largely unknown. We

24 built authorship social networks from publication records to test how sociality predicts

25 career progression and survival in a cohort of biologists contributing to three international

26 conferences in the 1990s. We show that sociality has the strongest effect for female

27 researchers but, regardless of gender, publishing with many diverse co-authors

28 significantly reduces time to become a principal investigator and increases career duration.

29 Publishing repeatedly with co-authors also enhances career progression in both genders,

30 but reduces career length for men. These findings demonstrate that the value of

31 collaboration extends beyond scientific advances, and can directly benefit the career

32 progression and longevity of research scientists themselves. Efforts to encourage

33 researchers at all levels to invest in collaborations, particularly with female researchers,

34 will help to close the gender gap in science and academia. 


\section{INTRODUCTION}

37 Surviving in academia as a scientist is widely recognised as a challenge, with researchers today experiencing greater job competition, prolonged job insecurity, and declines in

39 career length compared to 50 years ago ${ }^{1-7}$. For female scientists these challenges are 40 especially acute, since women suffer a variety of inequalities within science compared to 41 men ${ }^{8-16}$, causing them to leave academia more often, and earlier, than male scientists

$429,17,18$. Lack of diversity - of all kinds - has perverse outcomes, including hampering novel 43 insight and scientific advances ${ }^{19,20}$. Yet despite much effort to understand the underlying 44 causes (summarised in Fig. 1 of ${ }^{21}$ ), disparities between the genders show discouragingly

45 few signs of reducing $8,9,13,22-25$. Given these obstacles to career attainment and progression, 46 what lessons can we learn from scientists that have survived in science and carved out long careers for themselves?

One approach to this question is to consider studies that have investigated what underlies longevity and success, and to see if those qualities identified as important also apply to scientists and their careers. For social animals - which scientists surely are ${ }^{26-30}$

51 interacting with peers is a crucial component of lifetime fitness ${ }^{31-40}$ and lifespan ${ }^{41-43}$.

52 Several mechanisms have been proposed for how increased sociality could improve fitness and longevity, and that could be relevant to scientists and their careers. These include the role of group level cooperation in acquiring resources, social learning, and the attainment of social status ${ }^{37}$. In line with these ideas, scientists with larger research networks gain more funding ${ }^{44}$ and are more highly cited than scientists with fewer co-authors ${ }^{29,45-48}$,

57 while articles mentioned on social media gain more citations ${ }^{49,50}$, suggesting that sociality in science also promotes greater access to resources, knowledge of new research, and leads to higher status among scientific peers. In turn, funding and citation rates are both factors

60 that positively influence career progression and longevity in science ${ }^{51-54}$. The health 
61 benefits of sociality are also well-documented, and increased sociality is associated with

62 lower stress levels and reduced incidence of stress-related illness ${ }^{41,55-59}$. If a larger social

63 network helps researchers to better cope with the stresses associated with establishing and

64 maintaining a career in science and academia ${ }^{60-64}$, then we might also expect a positive

65 correlation between career lifespan and sociality, with less sociable individuals leaving

66 science earlier.

67 Here we apply the concepts of studies testing how sociality affects fitness and

survival to investigate how social strategies among scientists influence career advancement and duration. Understanding the connections between sociality and career progression and survival, and in particular how this might differ between genders, could shed light on social strategies that researchers may wish to follow, and provide greater understanding of the underlying causes of gender disparities in the sciences. Previous studies have demonstrated the value of research collaborations for academic success $\left({ }^{48,65-70}\right)$, and also

74 that social strategies may differ between the genders ${ }^{71-74}$. However, the survivability of scientific careers has attracted only limited attention ${ }^{1,75,76}$, and the links between sociality, gender, and survival in science are completely unexplored. We used social network analyses to quantify the structural properties of egocentric co-authorship networks constructed from the publication records (>53,000 papers) of over 900 gender-identified international scientists, publishing over almost four decades. In contrast to many previous 80 studies, we made no restrictions in terms of career stage ${ }^{72,74,77}$, journal ${ }^{69,78-80}$, or institute

$81{ }^{71}$, and included both researchers who subsequently left science, and those who are still active $^{76}$, allowing us to determine directly the effect of sociality on survival in science. 
study for two reasons: i) multi-authored papers are the norm in this field ${ }^{69}$, which is necessary for the construction of ego-centric networks, and ii) numerous studies of gender biases within ecology and evolutionary biology suggest that, while female researchers remain under-represented in senior or more visible roles ${ }^{25,81-85}$, biases against female 90 authors in terms of acceptance rates or editorial decisions are not readily apparent ${ }^{86-92}$ (but 91 see ${ }^{25}$ ). Thus, while this field certainly does not display gender equality, for the purposes of our publication-based study, gender bias in publishing is unlikely to be an issue. Importantly, since female researchers typically publish fewer papers than their male $71,76,77,79,80,93$,this study, we corrected the metrics we extracted from the social networks we constructed by controlling for variation in the number of papers that these focal authors published, thereby allowing us to account for the productivity gap between the genders.

We calculated three measures of author sociality from these publication-corrected social metrics: collaborativeness, which describes the number of unique co-authors a focal author publishes with; consistency, which describes how often, on average, a focal author

100 publishes with the same co-authors; and co-author connectedness, which describes how

101 often the same co-authors appear together across the publications of a focal author. We then used survival analyses (accelerated failure time models; AFT) to directly compare how sociality affects career progression and survival of female and male researchers, a major advancement on previous studies. We expected that scientists who were more social

105 in their approach to publishing - that is, were more collaborative, more consistent, and

106 who had co-authors that were less connected to each other- would i) be more likely to 107 become principal investigators (PIs), ii) become PIs more quickly, and iii) survive for longer in science. 


\section{RESULTS}

\section{Gender differences in publication output-corrected author sociality metrics and in}

\section{3 performance measures}

114 Our final dataset included 298 women (32\%) and 637 men (68\%), and three focal authors

115 for whom gender was unknown (see Supplementary methods S1 for more descriptive

116 statistics of the focal authors). We found that gender significantly affected

117 collaborativeness score (Generalised linear model [GLM], estimate \pm SE $=0.29 \pm 0.07$,

$118 t_{933}=4.21, p<0.001 ;$ Fig. S6D), and males were more collaborative than females (i.e. had

119 a greater number of co-authors on average, given their number of publications). By

120 contrast, gender did not affect consistency (GLM, estimate $\pm \mathrm{SE}=0.12 \pm 0.07, t_{933}=1.73$,

$121 p=0.08$; Fig. S6E) or co-author connectedness (GLM, estimate $\pm \mathrm{SE}=-2.00 \pm 1.40, t_{928}=$

$122-1.43, p=0.15$; Fig. S6F). Thus, with the exception of collaborativeness, we found no

123 evidence for underlying gender differences in how focal authors in our dataset published

124 their work in terms of their level of sociality. Gender differences were apparent, however,

125 in all three measures of academic performance that we investigated. Considering only the

126 effect of gender, male focal authors were significantly more likely to become PIs than their

127 female colleagues ( $63 \%$ of women versus $82 \%$ of men; GLM, estimate \pm SE $=0.97 \pm 0.16$,

$128 z .233=6.12, p<0.001)$ and did so in about $30 \%$ less time $($ AFT, estimate $=0.69[0.60$,

129 0.81]). Using the median time of eight years to reach PI status (based on all authors in our

130 dataset that did so), this means that, on average, female focal authors took just over six

131 months longer to reach this career stage than did male focal authors (restricted mean

132 survival time, between-group contrast: $\mathrm{M}-\mathrm{F}=-0.51$ years $[-0.84,-0.20], p=0.002$; Fig.

133 1B).

134 Being male also increased the likelihood of still being in science, on average, by

$13569 \%(\mathrm{AFT}$, estimate $=1.69[1.39,2.05])$. Thus, female focal authors had a mean $\pm \mathrm{SE}$ 
136 career length of $22.16 \pm 0.56$ years, while for men it was $25.90 \pm 0.34$ years. Over the

137 maximum 39-year career length of any focal author in our dataset, this equates to a female

138 focal author losing nearly 4.5 years of career time, on average, compared to a male focal

139 author (restricted mean survival time, between-group contrast: $\mathrm{M}-\mathrm{F}=4.48$ years [2.74,

$1406.23], p<0.001)$. This is a reduction in career length of $11 \%$ compared to male focal

141 authors (Fig. 1A).

\section{Effect of author sociality on PI status, time to PI, and career length}

\section{4 i) Likelihood of becoming a PI}

145 After accounting for gender, all three measures of authorship sociality significantly

146 predicted the likelihood of focal authors achieving PI status. In the case of

147 collaborativeness and consistency, the relationship was positive, such that focal authors

148 who did become PIs tended to have higher scores for these two author sociality metrics

149 (GLM, collaborativeness: estimate $\pm \mathrm{SE}=1.75 \pm 0.15, z 932=11.88, p<0.001$;

150 consistency: estimate $\pm \mathrm{SE}=0.39 \pm 0.09, z 932=4.33, p<0.001)$. The reverse was true for

151 co-author connectedness: focal authors with a lower connectedness score were more likely

152 to become PIs than those that had higher co-author connectedness scores (GLM, estimate \pm $\mathrm{SE}=-0.53 \pm 0.08, z 927=-6.33, p<0.001)$.

155 ii) Time to $\mathbf{P I}$

156 Collaborativeness significantly and positively influenced how long it took focal authors to

157 become PIs, but there was no effect of gender on this relationship (interaction term: AFT,

158 estimate $=1.17[0.99,1.38])$. For every increase of one in collaborativeness score, the time

159 to PI decreased by $21 \%$ (AFT, estimate $=0.79$ [0.73, 0.85]; Fig. 2A, B; Fig. 4).

160 Consistency was also a significant predictor of time to PI. Focal authors with higher 
161 consistency scores took significantly less time to become PIs, with the effect of

162 consistency being greater for female focal authors than for male focal authors (interaction

163 term: AFT, estimate $=1.34[1.14,1.58]$; female focal authors estimate $=0.64[0.55,0.74]$;

164 male focal authors estimate $=0.86$ [0.80, 0.93]; Fig. 2C, D; Fig. 4). Focal authors with

165 high co-author connectedness scores took longer to reach PI status than those with lower

166 scores, and this was dependent on gender (interaction term: AFT, estimate $=0.84[0.72$,

167 0.99]). An increase of one unit in co-author connectedness score decreased the time to PI

168 by approximately $37 \%$ in women (AFT, estimate $=1.37$ [1.16, 1. 60]; Fig. 2E; Fig. 4) but

169 only $13 \%$ in men (AFT, estimate = 1.13 [1.05, 1.22]; Fig. 2F; Fig. 4).

\section{Career length}

172 Collaborativeness positively predicted career length in both genders. Focal authors that

173 published with a greater number of co-authors (given their number of papers) were more

174 likely to continue publishing than focal authors with fewer co-authors than average (Fig.

$1753 \mathrm{~A}, \mathrm{~B})$. There was a significant interaction between this author sociality metric and gender,

176 with the effect of collaborativeness on career length being nearly double for female focal

177 authors compared to male focal authors (interaction term: AFT, estimate $=0.51[0.39$,

178 0.66]; gender-specific models: female focal authors, estimate $=3.93[3.16,4.89]$; male

179 focal authors, estimate $=2.33[2.02,2.68]$; Fig. 4). Thus, the impact of collaborativeness

180 on career length, as for time to PI, was greater for female focal authors than it was for their

181 male counterparts.

182 The effects of consistency (how frequently a focal author published with the same

183 co-authors, given their number of papers) differed between female and male focal authors

184 (interaction term: AFT, estimate $=0.77$ [0.64, 0.93]; Fig. 4). For female focal authors there

185 was a positive, but non-significant effect of consistency on career length, with increased 
consistency resulting in longer careers (AFT, estimate $=1.16[0.99,1.35]$; Fig. 3C). For

male focal authors, greater consistency negatively and significantly influenced career active in science compared to those with lower consistency scores. remaining in science by approximately $24 \%$ (Fig. 3E, F; Fig. 4). more pronounced effects for female focal authors than for their male counterparts (Fig. 4).

\section{Directionality of effect: Does publishing behaviour at early career stage predict}

\section{future academic survival?}

204 Network metrics extracted from focal authors based on their first ten years of publishing

205 showed very similar patterns in their relationship with scientific career survival to those

206 based on analysis of the entire dataset (Table S2). This was the case both for the direction

207 and the relative magnitude of any effect, and is in support of the hypothesis that network

208 metrics drive career length, rather than the other way round (see Methods: Does author

209 sociality affect scientific career length, or vice versa?). Extent of sociality during a focal

210 author's early career therefore predicted their future survival. Importantly however, in 
211 contrast to our findings based on the complete dataset, results for collaborativeness and co-

212 author connectedness were independent of gender. This implies that any differences in how

213 these two measures of author sociality affect the survival of female and male focal authors

214 only arise after their first ten years of publishing, which corresponds approximately to the

215 median time that focal authors in our dataset become PIs.

\section{DISCUSSION}

219 While the role of research collaborations in promoting publication output and citations has

220 been previously reported ${ }^{48,65-69}$, our study is the first to explicitly demonstrate the benefits of collaborative research to the progression and length of academic careers in science. Our findings reveal that scientists that collaborated more, had stronger connections to their coauthors, and that published with a wide diversity of relatively unconnected co-authors were more likely to become PIs, and became one more quickly. Being more collaborative and having less connected co-authors also increased the likelihood of remaining active in science for longer. Thus, the main finding of our study is that collaboration with a diverse community of co-authors is beneficial to career prospects and survival in science. The positive effects of collaborating early with eminent scientists have previously been reported ${ }^{70}$ but our study suggests that such benefits arise from collaboration in general,

230 and not only with leading scientists, or at early career stages.

232 productivity and career advancement ${ }^{8,71,72,76,77,79,80,93-95}$, we found that female researchers

233 published fewer papers (equivalent to a 58.5\% gender gap in productivity in our dataset),

234 were less likely to become a PI, took longer to become one, and had shorter careers on 235 average than male authors. Shorter career lengths and higher dropout rates explain a large 
236 proportion (but not all) of the productivity and impact differences that female researchers

237 suffer compared to their male colleagues ${ }^{76}$. Our study highlights how such negative effects

238 for women could be magnified: if reduced career length and lower productivity also restrict

239 opportunities for enhancing sociality by establishing new collaborations, then this would

240 further contribute to less favourable career outcomes for female academic scientists.

241 Female focal authors were less collaborative than men (i.e. had fewer unique co-

242 authors), as has been previously documented ${ }^{71,73,74,96}$ (but see ${ }^{72}$ ), and this result remained

243 after controlling for productivity differences between female and male focal authors (see

244 Methods: Accounting for the effect of publication number on social metrics). This is in

245 contrast to the findings of ${ }^{71}$, who found that the difference in co-author numbers between

246 genders could be fully explained by the lower publication rate and shorter career lengths of

247 women scientists. Nonetheless, collaborative style ${ }^{97-99}$ could be equally as important as

248 the number of collaborators for determining the value of collaborations to a researchers'

249 career. Furthermore, the publishing networks of female and male focal authors showed

250 similar levels of consistency and connectedness, suggesting that any inherent differences in

251 sociality between female and male researchers are likely small. Given this, a striking

252 finding of our study was that the effects of author sociality on career progression and

253 length were consistently stronger for female than for male focal authors. One explanation

254 for this may be that female researchers are judged more on the basis of whom they work

255 with than are male colleagues, making their collaborations all the more important ${ }^{22,100-102}$.

256 The gender composition of a researchers' network may also be more important for female

257 researchers than for male scientists. For example, more prestigious roles within

258 collaborative projects, including senior and corresponding authorships, are often assigned

259 to the detriment of women, potentially causing their contributions to research to be

260 undervalued ${ }^{103,104}$. Having a female senior author on a manuscript, however, increases 
261 both the overall proportion of women authors, and the probability of a female first author

$262{ }^{104}$. Similarly, having an inner social circle composed predominantly of women predicts

263 women's leadership success ${ }^{105}$. Alternatively, since researchers are more likely to publish

264 with colleagues of the same gender than is expected by chance ${ }^{99,106}$, gender preferences in

265 co-authors may further reinforce gender differences in career progression and length. This

266 effect could be further compounded since high-performing (and therefore ideal

267 collaborator ${ }^{102,107}$ ) male academics employ relatively fewer women, and thus provide

268 fewer associated collaboration opportunities ${ }^{24}$.

269 Consistency was the only measure of co-author sociality that had a negative effect

270 on any of the performance measures we assessed, and this only applied to career length for

271 male focal authors. Previous research indicates that the majority of collaborations between

272 researchers are weak and transitory ${ }^{108}$. However, those co-authors that a scientist

273 collaborates with repeatedly over their career - and that would therefore tend to raise the

274 consistency score of a focal author in our dataset - contribute significantly and positively

275 to above-average productivity and increased citation rates ${ }^{108}$. These are both factors that

276 would likely promote career advancement and length. Indeed, we found that higher

277 consistency positively influenced career progression in both genders. It is therefore unclear

278 why being more consistent in who you publish with should reduce career span for men,

279 particularly when there was no significant effect of consistency on career length for female

280 focal authors. 'Star' collaborators - those eminent scientists that stand out because of their

281 high funding rate, citation rate or prize-winning ability - can significantly enhance the

282 productivity and citation rate of their co-authors ${ }^{102,107}$. Closer investigation of exactly who

283 it is that female and male focal authors collaborate with, and how often, may shed more

284 light on this peculiar effect of consistency on career length. 
287 from ecological and evolutionary studies of sociality to understand survival in science.

288 Indeed, we find it intriguing that, despite building social networks based solely on

289 publication affiliations, our results show strong parallels with studies across a range of

290 species and contexts that investigate the role of sociality in enhancing performance and

291 longevity ${ }^{31,38,41,43}$. These studies often emphasise the importance of early life conditions

based on the first ten years of a focal authors' career were predictive of their overall career

294 length, suggesting that the approach to collaboration and publishing that a scientist takes

295 early on in their career has important implications for their future success ${ }^{70,111}$. Junior

scientists are typically more likely to be pursuers of collaborations, while senior

297 researchers are net attractors, having new collaborations proposed to them ${ }^{108}$. Our findings

298 suggest that for scientists early in their career, being an enthusiastic pursuer of

299 collaborations is beneficial in the long term, especially since the tendency to collaborate

300 increases with career length ${ }^{98}$, and how collaborative a scientist is predicts their

301 productivity better as their career progresses ${ }^{112}$. With the exception of consistency, we

302 found no effect of gender on how early career levels of sociality predicted career length, in

303 contrast to the clear gender asymmetry apparent in the entire dataset. We suggest that the

304 gender-specific effects of author sociality may arise only around the time that a researcher

305 becomes a PI.

Although our study focused specifically on contributors to International Society for

307 Behavioral Ecology conferences, the range of researchers that attend these conferences is

308 wide (see Methods: Identification of focal authors), and we expect our findings to be

309 applicable across ecology and evolutionary biology. Over the course of their careers, the 
310 researchers in our study published papers on a wide diversity of subjects in these

311 disciplines, and beyond. Furthermore, both the positive effects of sociality that we observe,

312 and the issues surrounding collaborative working, are unlikely to be subject-specific.

313 Regardless of discipline, finding and establishing new collaborations is not always

314 straightforward, and working more collaboratively may require overcoming barriers, such

315 as having a small peer network, or lacking access to funding ${ }^{113}$. This may be a particular

316 challenge for female researchers, who represent less of the workforce as seniority increases

$317{ }^{9}$, and especially since male researchers typically prefer to collaborate with other men ${ }^{99,106}$.

318 Researchers may also have concerns over the risk to reward ratio of forming new

319 collaborations ${ }^{113}$. A larger team of co-authors on a research project entails sharing any

320 reward for the work amongst a larger group of colleagues. Our findings imply that this

321 should not deter researchers from seeking out new collaborations. Rather, more

322 collaborators may increase the number of new networks that a scientist and their work can

323 be introduced to. Indeed, collaboration is frequently mentioned as an important factor in

324 scientists' own reflections on their success ${ }^{114}$ in 115 . In conclusion, our results suggest that

325 all researchers - but particularly those that are female - can enhance their career

326 progression and survival in science by collaborating widely and repeatedly. Creating

327 research environments that encourage collaboration - across disciplines and institutes,

328 among all career levels, and especially between the genders - will lead to greater and more

329 rapid scientific advances ${ }^{20}$, but can also assist in reducing the gender gap in science.

METHODS

\section{Identification of focal authors}

334 Our subjects were contributors to the International Society of Behavioral Ecology (ISBE) 
335 biennial meetings in 1992 (Princeton, USA, 766 participants), 1994 (Nottingham, UK, 521

336 participants) and 1996 (Canberra, Australia, 565 participants). ISBE conferences attract

337 researchers working in the field of behavioural ecology, but also in related areas including

338 animal behaviour, evolutionary biology, population biology, physiology, and molecular

339 biology (www.behavecol.com), and contributors therefore represent a broad spectrum of

340 research fields. We chose these three particular conferences because they occurred long

341 enough ago that any participants who were at the start of their career in the early 1990s

342 would by now have had sufficient time to reach PI status, should they have tried to do so

343 (how we define this is specified below). We also considered it likely that, particularly for

344 junior or early career researchers, for whom access to funding might be more limited, most

345 delegates would only be attending an international conference if they had a talk or poster to

346 present. Thus, contribution to a conference likely indicated active participation in science

347 at that time. Conferences are a common place for scientists to meet and find existing and

348 new collaborators ${ }^{116}$, and in the 1990s the ISBE conferences attracted many of the most

349 active researchers in behavioural ecology and related fields, and also many young students

350 embarking on a career in this field (pers. comm. Prof. Lotta Sundström and Prof. Nick

351 Davies, Feb. 2020). Finally, these conferences spanned three continents, thereby

352 maximising the global coverage of our sampling. Given these reasons, we are confident

353 that our initial study cohort captures as large and as representative a sample of the

354 behavioural ecology community at that time as we could hope to achieve.

355 A total of 1469 unique names were listed in the conference booklets for all three

356 conferences. There was no consistent format to how information on conference

357 participation was presented, and so all names were included in our initial pool of subjects,

358 regardless of whether they were listed as a delegate, a registrant, a participant, were

359 presenting a talk or a poster, or were a co-author on a presented talk or poster. Whilst our 
360 starting list may include some researchers who did not physically attend any of the

361 conferences (we have no way of knowing this), our aim was to include all those who may

362 nonetheless have contributed to the scientific content of these conferences in some way,

363 and thus could be identified as potentially being scientifically active. Our next steps in

364 refining this list (see 'Bibliometric procedures' and 'Restrictions', below) further identified

365 those who we considered to actually be active in science. Where possible, we recorded the

366 gender of conference contributors as male or female, based on their first name and/or

367 online profile, and noted the continent that they were associated with at the time of their

368 first conference registration. We then imported this list of individuals and associated

369 attributes into R version 3.5.0 ${ }^{117}$ in which all further data processing, analyses and

370 visualisations were carried out.

\section{Bibliometric procedures}

373 We used the 'rscopus' package ${ }^{118}$ to identify all those individuals in our starting pool of

374 conference participants that also existed in the academic database Scopus

375 (www.scopus.com), which claims to be "the largest abstract and citation database of peer-

376 reviewed literature". We filtered our list to only include those contributors that had a

377 Scopus Author Identifier (SAI), indicating that they had published at least one scientific

378 article. Subsequently, we manually searched Scopus by author name (first initial and

379 surname, gender anonymised), further restricting our dataset of potential authors to those

380 that had published at least one paper that could be considered as relevant to the field of

381 behavioural ecology, as judged from the title of the paper, the contents of the abstract, the

382 research specialisation(s) of the authors, and the journal of publication. We also combined

383 the publication records of any identified authors who had multiple Scopus profiles,

384 meaning that their output could be found through different SAIs. From our initial list of 
3851469 conference contributors, 108 did not have a SAI, suggesting that they had never

386 published a peer-reviewed paper. In a further 125 cases with multiple SAIs, it was not

387 possible to determine which was correct, either because an ambiguous author name yielded

388 too many search hits, and/or because it was unclear from the publication history. This left

389 us with a dataset of 1236 conference contributors who had all published at least once, and

390 whom we will now refer to as focal authors.

391 We downloaded the author list, title, publication year and journal of all publications

392 for all focal authors (as of September 2018) using the 'bibliometrix' package ${ }^{119}$, in

393 conjunction with Scopus API keys (www.api.elsevier.com) and manual searches (see

394 supplementary methods S2 for more information). To minimise the chance of name

395 synonymy - the same author appearing under slightly different names in our dataset - the

396 first name of each focal author in our dataset was reduced to the first initial, and any

397 accents were removed ${ }^{120}$. In four cases, two focal authors had the same surname and

398 initial, and were thus marked with a unique number to ensure they were recognised as

399 separate individuals in the analyses.

400

\section{$401 \quad$ Restrictions}

402 We applied several restrictions to our initial dataset of focal authors. We excluded focal

403 authors that only published single-author papers ( $\mathrm{n}=9$ authors), or had published fewer

404 than three papers in total ( $\mathrm{n}=91$ authors), as it was not possible to construct meaningful

405 social networks for these focal authors. We also excluded focal authors with more than 400

406 papers ( $\mathrm{n}=6$ authors), as they stood out as outliers (Fig. S1A). To produce a homogenous

407 cohort, and to minimise issues arising from changes in publishing patterns over time (e.g.

408 increasing numbers of co-authors on papers ${ }^{30,120-122}$ ) we further restricted our dataset to

409 those focal authors who published their first paper in 1980 or later (thereby excluding $\mathrm{n}=$ 
410192 authors; Fig. S1B). For those focal authors that remained in our dataset, we then

411 refined their output so that we only considered articles that were published in academic

412 journals. After manually correcting for errors or discrepancies in journal titles, the

413 following types of records were all excluded (6\% of total output, affecting 394 authors):

414 books and book chapters, trade journals, conference papers and proceedings, editorials,

415 errata, and short surveys. Finally, any articles with 20 or more authors (1\% of all papers in

416 the dataset; affecting 330 authors) were excluded from analyses, since these were extreme

417 outliers, and not representative of the general publishing norms within our dataset (Fig.

418 S1C). This left a final sample size of 938 focal authors with a total of 53,279 papers. We

419 checked whether any of these exclusions disproportionately affected either gender, given

420 the underlying proportion of female and male focal authors in our initial dataset. This was

421 not the case (see supplementary methods S3).

\section{Egocentric network construction}

424 Having defined our final dataset of focal authors we then generated an egocentric social

425 network for each focal author, based on their individual list of publications and co-authors.

426 Egocentric networks have the subject of interest (here, the focal author) as the central node

427 (the ego) ${ }^{123}$ and include connections to and among social contacts ('alters', or co-authors

428 in this case) that are adjusted by the strength of the association between ego and alter (here

429 equivalent to the number of co-authored papers that focal author and individual co-authors

430 share; Fig. S2) ${ }^{124}$. We calculated social network metrics by transforming the publication

431 record of each focal author into a binary 'group by ID' data structure with the 'asnipe'

432 package ${ }^{125}$, in which each row represented a paper, and every column was an author. This

433 was then converted into a weighted adjacency matrix in the 'asnipe' package, from which

434 we created weighted graphs with the 'igraph', 'intergraph' and 'network' packages ${ }^{126-128}$. 


\section{Egocentric network metrics}

436 We measured three metrics from each focal author's egocentric network. First, we

437 measured network size, or 'degree', which corresponds to the total number of co-authors in

438 the focal author's network. A higher degree indicates that an individual is associated with a

439 greater number of unique co-authors. Next, we extracted 'mean tie strength' (tie strength

440 hereafter), measured as the total number of times a focal author has published with her/his

441 co-authors across all their papers, divided by the number of unique co-authors. Tie strength

442 in this context thus provides an average measure of how many times a focal author

443 publishes with the same co-authors. Finally, we measured the 'global clustering

444 coefficient' of each focal author's egocentric network, using the transitivity function in the

445 'igraph' package ${ }^{126}$. This measure, ranging from zero to one, describes how connected, on

446 average, all authors in a focal author's network are, considering only the focal author's

447 papers ${ }^{129}$. A high connectedness - signified by a clustering coefficient close to one -

448 indicates that the same co-authors appear together on many of the focal author's

449 publications. This might be the case, for example, for a professor and her immediate group

450 of postdocs and $\mathrm{PhD}$ students. It is important to note that the clustering coefficient implies

451 nothing about how many papers co-authors may have published together without the focal

452 author. We could not calculate a clustering coefficient for five focal authors in our dataset

453 who had a maximum of one other co-author per publication.

\section{Accounting for the effect of publication number on social metrics}

456 Unsurprisingly, all three metrics were significantly influenced by how many publications a

457 focal author produced (degree: Generalised linear model (GLM), Poisson, estimate \pm SE $=$

$4580.009 \pm<0.001, z 936=233.4, p<0.001 ;$ tie strength: GLM, Gaussian, $\log 10$-transformed;

459 estimate $\pm \mathrm{SE}=0.002 \pm<0.001, t_{936}=13.85, p<0.001$; global clustering coefficient: 
460 GLM, Binomial; estimate $\pm \mathrm{SE}=-0.006 \pm<0.001, z_{931}=-595.5, p<0.001$; Fig. S3). That

461 is, focal authors with more papers tended to have more co-authors overall (a higher

462 degree), to publish more often with the same co-authors (a greater tie strength), and to have

463 co-authors that published less often with each other (a lower global clustering coefficient).

464 These effects were further compounded by clear gender differences in our dataset in terms

465 of the number of papers that female and male focal authors published. Overall, female

466 focal authors published significantly fewer papers than male focal authors (GLM, Poisson,

467 estimate $\pm \mathrm{SE}=0.54 \pm 0.01, z_{933}=50.81, p<0.001 ; \mathrm{F}: 38.46 \pm 2.30$ papers, median $=23.5$

468 papers, range: $3-234$ papers; M: $65.71 \pm 2.42$ papers, median $=51$ papers, range: $3-375$

469 papers), equivalent to a gender gap in productivity of $58.5 \%$. As a consequence, female

470 focal authors had significantly fewer co-authors (i.e. smaller networks and thus lower

471 degree values; GLM, $\log 10$-transformed, estimate $\pm \mathrm{SE}=0.57 \pm 0.08, t_{933}=6.88, p<$

4720.001 ; Fig. S6A), published with their co-authors less frequently (GLM, log10-

473 transformed, lower tie strength; estimate $\pm \mathrm{SE}=0.08 \pm 0.02, t_{933}=3.69, p<0.001$; Fig.

474 S6B), and published with co-authors that were less connected (global clustering

475 coefficient; GLM, binomial, estimate $\pm \mathrm{SE}=-0.30 \pm 0.002, \mathrm{z} 928=-170.7, p<0.001$; Fig.

476 S6C), compared to those of male focal authors. To account for these strong and gender-

477 biased effects of publication number, for each focal author we therefore regressed all three

478 metrics against their number of publications and used the residuals of these three models

479 (hereafter, degree residuals, strength residuals, and clustering residuals, respectively) for

480 all further analyses. All residuals were standardised by subtracting the mean and dividing

481 by the standard deviation.

We used degree residuals to define a focal author's 'collaborativeness', which can

483 be considered a measure of how many unique co-authors a focal author publishes with,

484 corrected for the number of papers they have published. Collaborativeness was analysed as 
485 a continuous variable. Thus, for a given number of published papers, a focal author with a

486 positive collaborativeness score can be considered to be more collaborative than the

487 average (i.e. publishes with a higher number of co-authors), while a focal author with a

488 negative collaborativeness score is less collaborative than the average.

489 We used degree residuals again, together with strength residuals, to obtain a

490 measure of how frequently a focal author published with the same co-author(s). We term

491 this 'consistency'. We extracted the residuals of a linear regression model with non-

492 constant variances ('Imvar' package ${ }^{130}$ ) that tested the effect of the degree residuals on the

493 strength residuals (estimate $\pm \mathrm{SE}=-0.36 \pm 0.02, \mathrm{z}_{4}=-15.60, p<0.001$ ). Consistency was

494 treated as a continuous variable. Focal authors with a positive consistency score published

495 more often with the same co-author(s) than the average for focal authors with the same

496 number of papers, and vice versa for those with a negative consistency score.

497 Finally, we used the clustering residuals to define the co-author connectedness of a 498 focal author. Focal authors with a positive co-author connectedness score publish with co-

499 authors that are more highly connected than the average for other focal authors with the

500 same number of papers. By contrast, a negative co-author connectedness score would

501 indicate that, compared to the average for focal authors with the same number of

502 publications, a focal author publishes with co-authors that have rarely or never published

503 with each other.

504 Thus, for each focal author, we ended up with three new metrics, adjusted for the

505 number of papers published, that describe how social they were in their approach to

506 publishing: their collaborativeness, their consistency, and their co-author connectedness.

508 Measures of academic performance and accounting for authorship name changes

509 We looked at academic performance in two ways: i) Following the definitions in ${ }^{94}$, we 
510 classified any focal author with at least three last-author publications as being a PI, and

511 measured time taken to reach PI status (time to PI) as the difference in years between their

512 first publication and their second last-author publication; ii) Scientific career length was

513 calculated as the time from first until last publication in our dataset ${ }^{1}$. For the purposes of

514 this study, we therefore consider a scientific career to be one that focuses on research and

515 publication, and that can be quantified solely according to the metric of publication output.

516 We fully recognise that there are other equally valid and important careers and means of

517 contributing to science that are not research-focused and that cannot be quantified in this

518 manner ${ }^{131}$. Our definition of career length relies on correctly identifying the publications

519 of focal authors. This task becomes more complicated if an author changes the name under

520 which they publish during their career, as might be the case particularly for female focal

521 authors (e.g. due to marriage). Unfortunately, there is little published work on how name

522 changes affect indexing and citation accuracy (but see ${ }^{132}$ for a notable exception), or how

523 indexing services cope with this issue. We checked whether author name changes could

524 have resulted in erroneous categorisation of a female focal author in our dataset as having

525 left science (i.e. stopped publishing; $\mathrm{n}=126$ ). To do this we manually searched the Internet

526 for any webpages that might contain publication lists for these female focal authors,

527 including Google Scholar and ResearchGate profiles, as well as professional and personal

528 webpages. We looked for any indication of a change in publishing name, as well as

529 checking the number of publications that a focal author had produced, and the date of their

530 last publication. We identified only two female focal authors who had changed their

531 publication name and thus were incorrectly assigned in our dataset as having left science.

532 The records for these focal authors were updated accordingly. 


\section{Statistical analyses}

\section{Data independence}

537 Whole social networks suffer from non-independence because metrics calculated for one

538 individual in a network are dependent on other members of the network ${ }^{133,134}$. If

539 unaccounted for, this may cause overestimation of true sampling variance, and

540 overconfidence in results ${ }^{133}$. Egocentric networks can also suffer from this problem if

541 many individuals occur across multiple networks. We tested the independence of our

542 egocentric networks by determining the proportion of networks in which focal authors also

543 appeared as co-authors, and the proportion of papers that consequently occurred in

544 multiple networks. Focal authors occurred on average in $<1 \%$ of networks as co-authors,

545 and in a maximum of 5.5\% (for one focal author only) of other networks (Fig. S4A).

546 Papers occurred, on average, in less than $1 \%$ of all networks (Fig. S4B). Thus, the

547 egocentric networks in this study are almost entirely independent of one another. Given

548 this extremely low level of overlap, we have followed the approach of ${ }^{134}$, and used

549 standard statistical procedures in our analyses.

\section{Effect of Gender}

552 We used general and generalised linear models to investigate how gender affected the

553 social metrics of interest after correcting for the number of papers published by each focal

554 author. We looked at how gender explained time to PI and career length by constructing

555 accelerated failure time (AFT) models, which are parametric survival models that provide

556 a robust statistical approach to analysing survival data. They are based upon the survival

557 curve, rather than the hazard function, as is the case for Cox proportional hazard models,

558 another commonly used approach for statistical modelling of survivorship data ${ }^{135}$. AFT

559 models provide an intuitive summary measure that describes the extent to which the 
560 survival curve is shifted forward or backward by the effect of the variable of interest. The

561 extent of this forward or backward shift is determined by the parameter $c$, also known as

562 the deceleration factor, in the relationship $S_{1}(c t)=S_{0}(t)$, where $S_{1}(t)$ is the survivorship of a

563 cohort receiving a particular 'treatment' at time $t$, while $S_{0}(t)$ is the survivorship of a

564 control cohort also at time $t$. If the 'treatment' (e.g. being more collaborative) increases

565 survival, this has the effect of shifting the survival curve forwards, as represented by a

566 value of $c>1{ }^{136}$. A confidence interval around $c$ can be calculated and, assuming that

567 similar models are built, the relative effect of different treatments on survival can be

568 compared. For an AFT approach to be valid, survival times are assumed to follow a

569 parametric distribution. All AFT models in this study were run with a lognormal

570 distribution, following visual examination of model fit, and comparison of log-likelihood and AIC values for models with different distributions. Residuals of AFT models were also examined for goodness of fit with the chosen distribution, and in all cases were found to be

573 a good fit. Validity of assumptions for normality and homoscedascity for linear models

574 were checked by visual inspection of residuals and normal probability plots. Results of

575 linear models are presented as mean \pm SE, unless stated otherwise, while we provide 576 estimates of the deceleration factor, with $95 \%$ confidence intervals, for the output of AFT

577 models, and with 'female' always set as the reference gender. Estimates from AFT models

578 with confidence intervals overlapping 1.0 are non-significant. We ran AFT models using

579 the functions survreg and flexsurvreg in the 'survival' and 'flexsurv' packages respectively

580137,138 . We used the 'Ime4' package ${ }^{139}$ to run general and generalised linear models.

\section{Effect of author sociality on time to PI, and career length}

583 We ran AFT models to evaluate whether collaborativeness, consistency and co-author

584 connectedness predicted time to PI and career duration. We included gender, and the 
585 interaction between gender and the social metric of interest, in all models. Where there was

586 a significant interaction between gender and a measure of author sociality, we re-ran

587 models for each gender separately. When this interaction was not significant, models were

588 re-run without the interaction term to obtain an estimate for the relevant measure of author

589 sociality. We also calculated restricted mean survival times using the 'survRM2' package

$590 \quad{ }^{140}$. A restricted mean survival time is a measure of average survival up to a specific time

591 point. It is calculated from a survival curve by determining the area under the curve (AUC)

592 and then comparing the AUCs for different survival curves (for example, to examine

593 gender differences in survival up to a certain time ${ }^{141}$ ).

594 In a first set of survival models we calculated the fraction of focal authors that

595 became a PI within $x$ years since their first publication year. Seven hundred and eight focal

596 authors became PI (i.e. published at least three last-author papers) before the end of our

597 sampling period (2018), leaving 230 focal authors (25\%) that did not, and were therefore

598 censored in this analysis. Eighteen authors produced their second last-author paper in the

599 same year as their first paper, and because survival models would otherwise ignore these

600 zero values, these were given dummy values of 0.5 years. Re-running survival analyses

601 with these authors excluded from the dataset did not alter the outcome of our models. We

602 ran binomial logistic regression models to ascertain the role of author sociality in the

603 likelihood of focal authors becoming PIs.

604 In a second set of survival models we investigated the effects of the network

605 metrics on focal authors' career length (number of years publishing). The survival curves

606 were calculated as the fraction of focal authors still publishing after $x$ years since their first

607 publication year. We assumed focal authors to have stopped publishing, and thus, from the

608 perspective of this study, for their scientific career to have ended, if at least two years had

609 elapsed since their last publication. This cut-off was chosen as the majority (80\%) of 
610 publication gaps - years in which a focal author published no papers - were shorter than

611 two years (Fig. S5). Note that posthumous publishing (i.e. publications that include the

612 name of an author who is no longer alive) is not an issue for our analyses as such

613 individuals still continued to contribute to science. Focal authors that were publishing in

6142016 or later ( $68 \%$ of authors) were assumed to still be active, and were censored in the

615 analyses. Female focal authors might have longer or more frequent career gaps if, on

616 average, they are more likely to take time away from their careers than do male focal

617 authors, for example due to maternity leave or extended child care duties. To account for

618 this possibility, we repeated our analyses while taking a more conservative censoring

619 approach that likely overestimated the number of censored subjects (still publishing in

6202012 and 2014, respectively, and thus allowing for a gap between publications of up to 6

621 years). All analyses yielded highly comparable results (Table S1). We also determined

622 whether the severity of our censoring approach (still publishing in 2012, 2014 or 2016)

623 disproportionately affected our measure of the number of female or male focal authors that

624 were considered to have left science. It did not (Supplementary methods S3).

626 Does author sociality affect scientific career length, or vice versa?

627 A positive correlation between any our three measures of author sociality and career length

628 might arise if focal authors that have longer careers, or are in more senior positions in

629 science, are deemed as more attractive to collaborate with, or have simply had more time

630 to establish successful and enduring collaborations. To test the directionality of any

631 relationship between our measures of author sociality and career survival we investigated

632 whether a focal authors' publishing behaviour in their early career predicted their future

633 academic survival. If similar patterns arise between network metrics calculated at the early

634 career stage, before focal authors have had time to establish their prominence and future 
635 academic survival, then we argue that this provides strong evidence that it is the network

636 metrics measured here that drive career length, and not the other way round. To investigate

637 this, we conducted a separate analysis of focal authors that started publishing the year prior

638 to their first attendance at an ISBE conference or later (to produce a homogeneous cohort),

639 and calculated the three author sociality metrics for up to their first ten publication years ( $n$

$640=224$ authors; $33.9 \%$ women; 3675 publications). We then ran AFT regression models to

641 test the effects of network metric, gender, and their interaction, on career length, as

642 previously described.

643

644 Data and code availability

645 The data that support the findings of this study, in which focal author names are

646 anonymised, will be available via Open Science Framework. Scripts for computational

647 analysis of this data will also be available via Open Science Framework. We will also

648 provide the code used to access publications available on Scopus for any given Scopus

649 Author Identifier (SAI), as well as code for data wrangling, calculation of social network

650 metrics and academic performance measures. For purposes of confidentiality, the names

651 and SAIs of focal authors used in this study will not be made available. For reviewing

652 purposes, data and code are available here: tiny.cc/vanderwal.

653

654 


\section{ACKNOWLEDGEMENTS}

656 We thank Neeltje Boogert, Alecia Carter, and Hannah Rowland for initial discussions

657 about the project, Damien Farine for advice on early analyses, Wendy King for access to

658 the ISBE archives, Rahia Mashoodh for assistance with dredging the Scopus API, Lauren

659 Brent for advice with combining network and survival analyses, Chris Duncan for

660 suggesting the use of AFT models, and Nick Davies, Lotta Sundström, and the Evolution,

661 Sociality and Behaviour group at the University of Helsinki for valuable discussions about

662 publishing, the history of behavioural ecology, and our results. J.E.M.vdW. was supported

663 by a grant from the Association for the Study of Animal Behaviour (ASAB) awarded to

664 R.T. R.T. was supported by an Independent Research Fellowship from the Natural

665 Environment Research Council UK (grant number NE/K00929X/1) and a start-up grant

666 from the Helsinki Institute of Life Science (HiLIFE), University of Helsinki. N.P.C.H. was

667 supported by a Leverhulme Early Career Fellowship from the Leverhulme Trust.

668

669 AUTHOR CONTRIBUTIONS

670 N.P.C.H. and R.T. conceived the study and all authors developed the concept. All authors

671 collected the data. J.E.M.vdW. prepared the data for analysis and ran the social network

672 analyses. N.P.C.H. carried out the AFT survival analyses. N.P.C.H. and J.E.M.vdW.

673 prepared the initial draft and all authors edited and approved the manuscript. 


\section{REFERENCES}

675 1. Milojević, S., Radicchi, F. \& Walsh, J. P. Changing demographics of scientific

676 careers: the rise of the temporary workforce. Proc. Natl. Acad. Sci. 115, 201800478

$677 \quad$ (2018).

678 2. Marshall, J., Dennehy, J., Harris, D. J. \& Mika, M. Letter to the editors: in the academic job market, will you be competitive? a case study in ecology and evolutionary biology. Isr. J. Ecol. Evol. 55, 381-392 (2009).

3. Daniels, R. J. A generation at risk: young investigators and the future of the biomedical workforce. Proc. Natl. Acad. Sci. 112, 313-318 (2015).

4. Alberts, B., Kirschner, M. W., Tilghman, S. \& Varmus, H. Rescuing US biomedical research from its systemic flaws. Proc. Natl. Acad. Sci. 111, 5773-5777 (2014).

5. Fang, F. C. \& Cadadevall, A. Competitive science: is competition ruining science? Infect. Immun. 83, 1229-1234 (2015).

6. Wellcome Trust. What Researchers Think About the Culture They Work In. (2020).

7. Smith, D. K. The race to the bottom and the route to the top. Nat. Chem. 12,101$103(2020)$.

8. Larivière, V., Ni, C., Gingras, Y., Cronin, B. \& Sugimoto, C. R. Bibliometrics: global gender disparities in science. Nature 504, 211-213 (2013).

9. Holman, L., Stuart-fox, D. \& Hauser, C. E. The gender gap in science: how long until women are equally represented? PLoS Biol. 16, e2004956 (2018).

10. Long, J. S., Allison, P. D. \& McGinnis, R. Rank advancement in academic careers: sex differences and the effects of productivity. Am. Sociol. Rev. 58, 703-722 (1993). 
699 12. Pohlhaus, J. R., Jiang, H., Wagner, R. M., Schaffer, W. T. \& Pinn, V. W. Sex differences in application, success, and funding rates for NIH extramural programs. Acad. Med. 86, 759-767 (2011).

13. Brower, A. \& James, A. Research performance and age explain less than half of the gender pay gap in New Zealand universities. PLoS One 15, e0226392 (2020).

14. Helmer, M., Schottdorf, M. \& Neef, A. Gender bias in scholarly peer review. Elife e21718 (2017). doi:10.7554/eLife.21718

706

15. Lee, R. Van Der \& Ellemers, N. Gender contributes to personal research funding success in The Netherlands. Proc. Natl. Acad. Sci. U. S. A. 112, 12349-12353 (2015).

16. West, J. D., Jacquet, J., King, M. M., Correll, S. J. \& Bergstrom, C. T. The role of gender in scholarly authorship. PLoS One 8, e66212 (2013).

711 17. United Nations Educational, S. and C. O. UNESCO Science Report: Towards 2030. (2015).

18. Blickenstaff, J. C. Women and science careers: leaky pipeline or gender filter? Gend. Educ. 17, 369-386 (2005).

19. Hong, L. \& Page, S. E. Groups of diverse problem solvers can outperform groups of high-ability problem solvers. Proc. Natl. Acad. Sci. 101, 16385-16389 (2004).

20. Nielsen, M. W. et al. Gender diversity leads to better science. Proc. Natl. Acad. Sci. 114, 1740-1743 (2017).

719 21. Grogan, K. E. How the entire scientific community can confront gender bias in the workplace. Nat. Ecol. Evol. 3, 3-6 (2019). national funding agency Holly. Lancet 393, 531-540 (2019). 
23. Lundine, J., Bourgeault, I. L., Clark, J., Heidari, S. \& Balabanova, D. The gendered system of academic publishing. Lancet 391, 1754-1756 (2018).

24. Sheltzer, J. M. \& Smith, J. C. Elite male faculty in the life sciences employ fewer women. Proc. Natl. Acad. Sci. 111, 10107-10112 (2014).

25. Fox, C. W. \& Paine, C. E. T. Gender differences in peer review outcomes and manuscript impact at six journals of ecology and evolution. Ecol. Evol. 9, 35993619 (2019).

26. Hunter, L. \& Leahey, E. Collaborative research in sociology: trends and contributing factors. Am. Sociol. 39, 290-306 (2008).

27. Milojević, S. Principles of scientific research team formation and evolution. Proc. Natl. Acad. Sci. 111, 3984-3989 (2014).

28. Weeks, W. B., Wallace, A. E. \& Kimberly, B. C. S. Changes in authorship patterns in prestigious US medical journals. Soc. Sci. Med. 59, 1949-1954 (2004).

29. Wuchty, S., Jones, B. F. \& Uzzi, B. The increasing dominance of teams in production of knowledge. Science 316, 1036-1038 (2007).

30. Barlow, J. et al. On the extinction of the single-authored paper: The causes and consequences of increasingly collaborative applied ecological research. J. Appl. Ecol. 55, 1-4 (2018).

31. Silk, J. B., Alberts, S. C. \& Altmann, J. Social bonds of female savanna baboons enhance infant survival. Science 302, 1231-1234 (2003).

32. Royle, N. J., Pike, T. W., Heeb, P., Richner, H. \& Kölliker, M. Offspring social network structure predicts fitness in families. Proc. R. Soc. B Biol. Sci. 279, 4914 4922 (2012).

747 33. Stanton, M. A. \& Mann, J. Early social networks predict survival in wild bottlenose dolphins. PLoS One 7, 1-6 (2012). 
749 34. Lehmann, J., Majolo, B. \& McFarland, R. The effects of social network position on the survival of wild Barbary macaques, Macaca sylvanus. Behav. Ecol. 27, 20-28 (2016).

35. Brent, L. J. N. \& Platt, M. L. Family network size and survival across the lifespan of female macaques. Proc. R. Soc. B Biol. Sci. 284, 20170515 (2017). support in pregnancy: psychosocial correlates of birth outcomes and postpartum depression. J. Pers. Soc. Psychol. 65, 1243-1258 (1993).

37. Thompson, N. A. Understanding the links between social ties and fitness over the life cycle in primates. Behaviour 156, 859-908 (2019).

38. Cameron, E. Z., Setsaas, T. H. \& Linklater, W. L. Social bonds between unrelated females increase reproductive success in feral horses. Proc. Natl. Acad. Sci. 106, 13850-13853 (2009).

39. Silk, J. B. The adaptive value of sociality in mammalian groups. Philos. Trans. $R$. Soc. B Biol. Sci. 362, 539-559 (2007).

40. Berkman, L. F., Melchior, M., Chastang, J., Niedhammer, I. \& Goldberg, M. Social integration and mortality: a prospective study of French employees of electricity of France-Gas of France. Am. J. Epidemiol. 159, 167-174 (2004).

41. Holt-Lunstad, J., Smith, T. B. \& Layton, J. B. Social relationships and mortality risk: a meta-analytic review. PLoS Med. 7, e1000316 (2010). 241, 540-545 (1971).

43. Archie, E. A. et al. Social affiliation matters: both same-sex and opposite-sex relationships predict survival in wild female baboons. Proc. R. Soc. B 281, 
44. Ebadi, A. \& Schiffauerova, A. How to receive more funding for your research? Get connected to the right people! PLoS One 10, e0133061 (2015).

45. Didegah, F. \& Thelwall, M. Which factors help authors produce the highest impact research? Collaboration, journal and document properties. J. Informetr. 7, 861-873 (2013).

46. Abbasi, A., Chung, K. S. K. \& Hossain, L. Egocentric analysis of co-authorship network structure, position and performance. Inf. Process. Manag. 48, 671-679 (2012).

47. Ronda-Pupo, G. A. \& Katz, J. S. The power law relationship between citation impact and multi-authorship patterns in articles in Information Science \& Library Science journals. Scientometrics 114, 919-932 (2018).

48. Li, E. Y., Liao, C. H. \& Yen, R. H. Co-authorship networks and research impact: a social capital perspective. Res. Policy 42, 1515-1530 (2013).

49. Lamb, C. T., Gilbert, S. L. \& Ford, A. T. Tweet success? Scientific communication correlates with increased citations in Ecology and Conservation. PeerJ 6, e4564 (2018).

50. Finch, T., O’Hanlon, N. \& Dudley, S. P. Tweeting birds: online mentions predict future citations in ornithology. R. Soc. Open Sci. 4, 171371 (2017).

51. Godin, B. The impact of research grants on the productivity and quality of scientific research. (2003).

52. Bol, T., Vaan, M. De \& Rijt, A. Van De. The Matthew effect in science funding. Proc. Natl. Acad. Sci. 115, 4887-4890 (2018).

53. Petersen, A. M., Jung, W.-S., Yang, J.-S. \& Stanley, H. E. Quantitative and empirical demonstration of the Matthew effect in a study of career longevity. Proc. Natl. Acad. Sci. 108, 18-23 (2011). 
799 54. Mazloumian, A., Eom, Y., Helbing, D., Lozano, S. \& Fortunato, S. How citation boosts promote scientific paradigm shifts and Nobel prizes. PLoS One 6, e18975

801 (2011).

802

55. Wittig, R. M. et al. Social support reduces stress hormone levels in wild chimpanzees across stressful events and everyday affiliations. Nat. Commun. 7, $13361(2016)$.

56. Hennessy, M. B., Kaiser, S. \& Sachser, N. Social buffering of the stress response: diversity, mechanisms, and functions. Front. Neuroendocrinol. 30, 470-482 (2009).

57. Kikusui, T., Winslow, J. T. \& Mori, Y. Social buffering: relief from stress and anxiety. Philos. Trans. R. Soc. B Biol. Sci. 361, 2215-2228 (2006).

58. Wittig, R. M. et al. Focused grooming networks and stress alleviation in wild female baboons. Horm. Behav. 54, 170-177 (2008).

811 59. Alberts, S. C. \& Altmann, J. Hypercortisolism associated with social subordiance or social isolation among wild baboons. Arch. Gen. Psychiatry 54, 1137 (1997).

60. Herbert, D. L., Coveney, J., Clarke, P., Graves, N. \& Barnett, A. G. The impact of funding deadlines on personal workloads, stress and family relationships: a qualitative study of Australian researchers. BMJ Open 4, e004462 (2014).

61. Woolston, C. PhDs: the tortuous truth. Nature 575, 403-406 (2017).

817 62. O’Brien, T. \& Guiney, G. Staff Wellbeing in Higher Education. (2018).

818 63. Kinman, G., Jones, F., Kinman, G. \& Jones, F. A life beyond work? Job demands, 819 work-life balance, and wellbeing in UK academics. J. Hum. Behav. Soc. Environ. 17, 41-60 (2008).

64. Powell, K. Young, talented and fed-up: scientists tell their stories. Nature 538, 446449 (2016).

823 65. Bidault, F. \& Hildebrand, T. The distribution of partnership returns: evidence from 
824 co-authorships in economics journals. Res. Policy 43, 1002-1013 (2014).

825 66. Lee, S. \& Bozeman, B. The impact of research collaboration on scientific

826 productivity. Soc. Stud. Sci. 35/5(October 35, 673-702 (2005).

827 67. Ductor, L. Does co-authorship lead to higher academic productivity? Oxf. Bull.

$828 \quad$ Econ. Stat. 77, 0305-9049 (2015).

829 68. He, Z., Geng, X. \& Campbell-hunt, C. Research collaboration and research output: a 830 longitudinal study of 65 biomedical scientists in a New Zealand university. Res.

$831 \quad$ Policy 38, 306-317 (2009).

832 69. Pike, T. W. Collaboration networks and scientific impact among behavioral 833 ecologists. Behav. Ecol. 2, 431-435 (2010).

834 70. Li, W., Aste, T., Caccioli, F. \& Livan, G. Early coauthorship with top scientists predicts success in academic careers. Nat. Commun. 10, 1-9 (2019).

836 71. Zeng, X. H. T. et al. Differences in collaboration patterns across discipline, career stage, and gender. PLoS Biol. 14, e1002573 (2016).

838 72. Bozeman, B. \& Gaughan, M. How do men and women differ in research 839 collaborations? An analysis of the collaborative motives and strategies of academic researchers. Res. Policy 40, 1393-1402 (2011).

841 73. Abramo, G., Andrea, C., Angelo, D. \& Murgia, G. Gender differences in research collaboration. J. Informetr. 7, 811-822 (2013).

843 74. Larivière, V., Vignola-Gagné, E., Villeneuve, C., Gélinas, P. \& Gingras, Y. Sex 844 differences in research funding, productivity and impact: an analysis of Québec

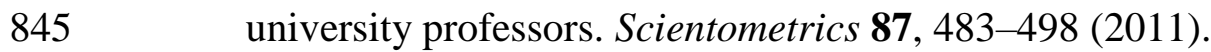

846 75. Kaminski, D. \& Geisler, C. Survival analysis of faculty retention in science and 847 engineering by gender. Science 335, 864-866 (2012).

848 76. Huang, J., Gates, A. J., Sinatra, R. \& Barabási, A.-L. Historical comparison of 
gender inequality in scientific careers across countries and disciplines. Proc. Natl.

851 77. Long, J. S. Measures of sex differences in scientific productivity. Soc. Forces 71, 159-178 (1992).

78. Tajedini, O., Ghazizade, A. \& Sadatmoosavi, A. Identifying the effects of coauthorship strategies on the citation-based performance of scholars: a social networks analysis. J. Scientometr. Res. 7, 19-28 (2018).

79. Teele, D. L. \& Thelen, K. Gender in the journals: publication patterns in political science. Polit. Sci. Polit. 50, 433-447 (2017).

80. Tower, G., Plummer, J. \& Ridgewell, B. A multidisciplinary study of gender-based research productivity in the worlds best journals. J. Divers. Manag. 2, 23-32 (2007).

81. Martin, L. J. Where are the women in ecology? Front. Ecol. Environ. 10, 177-178 (2012).

82. Schroeder, J. et al. Fewer invited talks by women in evolutionary biology symposia. J. Evol. Biol. 26, 2063-2069 (2013).

83. Damschen, E. I. et al. Visibility matters: increasing knowledge of women's contributions to ecology. Front. Ecol. Environ. 3, 212-219 (2005).

84. Whelan, A. M. \& Schimel, D. S. Authorship and Gender in ESA Journals. Bull. Ecol. Soc. Am. 100, 1-9 (2019).

85. Farr, C. M. et al. Addressing the Gender Gap in Distinguished Speakers at Professional Ecology Conferences. Bioscience 67, 464-468 (2017).

86. Edwards, H. A., Schroeder, J. \& Dugdale, H. L. Gender differences in authorships e0201725 (2018).

873 87. Borsuk, R. M., Budden, A. E., Leimu, R., Aarssen, L. W. \& Lortie, C. J. The 
influence of author gender, national language and number of authors on citation rate in rcology. Open Ecol. J. 2, 25-28 (2009).

88. Tregenza, T. Gender bias in the refereeing process? Trends Ecol. Evol. 17, 349-350 (2002).

89. Buckley, H. L., Sciligo, A. R., Adair, K. L., Case, B. S. \& Monks, J. M. Is there gender bias in reviewer selection and publication success rates for the New Zealand Journal of Ecology? N. Z. J. Ecol. 38, 335-339 (2014).

90. Primack, R. B., Ellwood, E., Miller-rushing, A. J., Marrs, R. \& Mulligan, A. Do gender, nationality, or academic age affect review decisions? An analysis of submissions to the journal Biological Conservation. Biol. Conserv. 142, 2415-2418 (2009).

91. Fox, C. W., Burns, C. S. \& Meyer, J. A. Editor and reviewer gender in fl uence the peer review process but not peer review outcomes at an ecology. Funct. Ecol. 30, 140-153 (2016).

92. Fox, C. W., Burns, C. S., Muncy, A. D. \& Meyer, J. A. Gender differences in patterns of authorship do not affect peer review outcomes at an ecology journal. Funct. Ecol. 30, 126-139 (2016).

93. Djupe, P. A., Smith, A. E. \& Sokhey, A. E. Explaining gender in the journals: how submission practices affect publication patterns in political science. Polit. Sci. Polit. 52, (2019).

894 94. van Dijk, D., Manor, O. \& Carey, L. B. Publication metrics and success on the academic job market. Curr. Biol. 24, R516-R517 (2014). 
899 96. Bozeman, B. \& Corley, E. Scientists' collaboration strategies: implications for

900 scientific and technical human capital. Res. Policy 33, 599-616 (2004).

901 97. Rhoten, D. \& Pfirman, S. Women in interdisciplinary science: exploring preferences 902 and consequences. Res. Policy 36, 56-75 (2007).

903 98. Van Rijnsoever, F. J. \& Hessels, L. K. Factors associated with disciplinary and 904 interdisciplinary research collaboration. Res. Policy 40, 463-472 (2011).

905 99. Araújo, E. B., Araújo, N. A. M., Moreira, A. A., Herrmann, H. J. \& Andrade Jr., S. 906 Gender differences in scientific collaborations: women are more egalitarian than men. PLoS One 12, e0176791 (2017).

908 100. Wennerås, C. \& Wold, A. Nepotism and sexism in peer-review. Nature 387, 341343 (1997).

910 101. Kaatz, A., Gutierrez, B. \& Carnes, M. Threats to objectivity in peer review: the case 911 of gender. Trends Pharmacol Sci. 35, 371-373 (2014).

912 102. Borjas, G. J. \& Doran, K. B. Which peers matter? The relative impacts of 913 collaborators, colleagues, and competitors. Rev. Econ. Stat. 97, 1104-1117 (2015).

914 103. Macaluso, B., Larivière, V. \& Sugimoto, T. Is science built on the shoulders of women? A study of gender differences in contributorship. Acad. Med. 91, 1136-

916 1142 (2016).

917 104. Fox, C. W., Ritchey, J. P. \& Paine, C. E. T. Patterns of authorship in ecology and evolution: first, last, and corresponding authorship vary with gender and geography.

920 105. Yang, Y., Chawla, N. V \& Uzzi, B. A network's gender composition and communication pattern predict women's leadership success. Proc. Natl. Acad. Sci. 116, 2033-2038 (2019).

923 106. Holman, L. \& Morandin, C. Researchers collaborate with same-gendered colleagues 
more often than expected across the life sciences. PLoS One 14, e0216128 (2019).

925

926

927

928

929

930

931

932

933

934

935

936

937

938

939

940

941

942

943

944

945

946

947

948

107. Azoulay, P., Graff Zivin, J. S. \& Wang, J. Superstar extinction. Q. J. Econ. 125, 549-589 (2010).

108. Petersen, A. M. Quantifying the impact of weak, strong, and super ties in scientific careers. Proc. Natl. Acad. Sci. 112, E4671-E4680 (2015).

109. Mann, J., Stanton, M. a., Patterson, E. M., Bienenstock, E. J. \& Singh, L. O. Social networks reveal cultural behaviour in tool-using using dolphins. Nat. Commun. $\mathbf{3}$, 980 (2012).

110. Laurance, W. F., Useche, D. C., Laurance, S. G. \& Bradshaw, C. J. A. Predicting publication success for biologists. Bioscience 63, 817-823 (2013).

111. Qi, M., Zeng, A., Li, M., Fan, Y. \& Di, Z. Standing on the shoulders of giants: the effect of outstanding scientists on young collaborators' careers. Scientometrics 111, $1839-1850$ (2017).

112. Hu, Z., Chen, C. \& Liu, Z. How are collaboration and productivity correlated at various career stages of scientists? Scientometrics 101, 1553-1564 (2014).

113. Pannell, J. L. et al. An early career perspective on encouraging collaborative and interdisciplinary research in ecology. Ecosphere 10, e02899 (2019).

114. Leahey, E. \& Cain, C. L. Straight from the source: accounting for scientific success. Soc. Stud. Sci. 43, 927-951 (2013).

115. Parish, A. J., Boyack, K. W. \& Ioannidis, J. P. A. Dynamics of co-authorship and productivity across different fields of scientific research. PLoS One 13, 1-12 (2018).

116. Campos, R., Leon, F. \& McQuillin, B. Lost in the storm: the academic collaborations that went missing in hurricane ISSAC. Econ. J. 128, 995-1018 (2018).

117. R Core Team. R: a language and environment for statistical computing. (2018). 
949 118. Muschelli, J. Gathering bibliometric information from the Scopus API using

950 rscopus. $R$ Journal (2018).

951 119. Aria, M. \& Cuccurullo, C. bibliometrix: An R-tool for comprehensive science mapping analysis. J. Informetr. 11, 959-975 (2017).

953

120. Arnaboldi, V., Dunbar, R. I. M., Passarella, A. \& Conti, M. Analysis of coauthorship ego networks. J. Commun. Dis. 9564, (2016).

121. Perez, T. M. \& Hogan, J. A. The changing nature of collaboration in tropical ecology and conservation. Biotropica 50, 563-567 (2018).

122. Fanelli, D. \& Larivière, V. Researchers' individual publication rate has not increased in a century. PLoS One 11, e0149504 (2016).

123. Crossley, B. N. et al. Social network analysis for ego-nets. (SAGE Publications Ltd, 960 2015).

124. Newman, M. E. J. Scientific collaboration networks. I. Network construction and fundamental results. Phys. Rev. E 64, 8 (2001).

125. Farine, D. R. Animal social network inference and permutations for ecologists in R using asnipe. Methods Ecol. Evol. 4, 1187-1194 (2013).

126. Csárdi, G. \& Nepusz, T. The igraph software package for complex network research. InterJournal (2006). doi:10.3724/SP.J.1087.2009.02191

127. Bojanowski, M. intergraph: coercion routines for network data object. Retrieved from http://mbojan.github.io/intergraph (2015).

969 128. Butts, C. T. network: a package for managing relational data in R. J. Stat. Softw. 24, $1-36(2008)$.

129. Prokhorenkova, L. O. \& Samosvat, E. Global clustering coefficient in scale-free networks. arXiv (2014). doi:10.1007/978-3-642-30541-2

973 130. Partners Posthuma. lmvar: linear regression with non-constant variances. Retrieved 
from https://CRAN.R-project.org/package= $(2018)$

975

131. Hanlon, S. M. Scientists who leave research to pursue other careers in science are

976 still scientists. Proc. Natl. Acad. Sci. 116, 17624 (2019).

977

132. Pellack, L. J. \& Kappmeyer, L. O. The ripple effect of women's name changes in indexing, citation, and authority control. J. Am. Soc. Inf. Sci. Technol. 62, 440-448

979 (2011).

980

133. Rumsey, A. R. The Association Between Co-authorship Network Structures and Successful Academic Publishing Among Higher Education Scholars. PhD thesis

982 (2006).

983

134. Turner, J. W., Bills, P. S., Holekamp, K. E. \& Turner, J. W. Ontogenetic change in determinants of social network position in the spotted hyena. Behav. Eclogy

985 Sociobiol. 72, 1-15 (2018).

986

135. Wei, L. J. The accelerated failure time model: a useful alternative to the cox regression model in survival analysis. Stat. Medice 11, 1871-1879 (1992).

136. Swindell, W. R. Accelerated failure time models provide a useful statistical framework for aging researcg. Exp. Gerontol. 44, 190-200 (2010).

990

137. Therneau, T. M., Crowson, C. \& Atkinson, E. Using time dependent covariates and 991 time dependent coefficients in the cox model. Survival Vignettes 1-27 (2016).

138. Jackson, C. flexsurv: a platform for parametric survival modeling in R. J. Stat. Softw. 70, 1-33 (2016).

994 139. Bates, D., Mächler, M. \& Walker, S. C. Fitting Linear Mixed-Effects Models Using $995 \quad$ lme4. J. Stat. Softw. 67, (2015). restricted mean survival time. 1-7 (2017).

998 141. Royston, P. \& Parmar, M. K. B. Restricted mean survival time: an alternative to the 
bioRxiv preprint doi: https://doi.org/10.1101/2020.03.02.973479; this version posted March 4, 2020. The copyright holder for this preprint (which was not certified by peer review) is the author/funder, who has granted bioRxiv a license to display the preprint in perpetuity. It is made available under aCC-BY-NC-ND 4.0 International license. outcome. BMC Med. Res. Methodol. 13, 2409-2421 (2013).

1001 

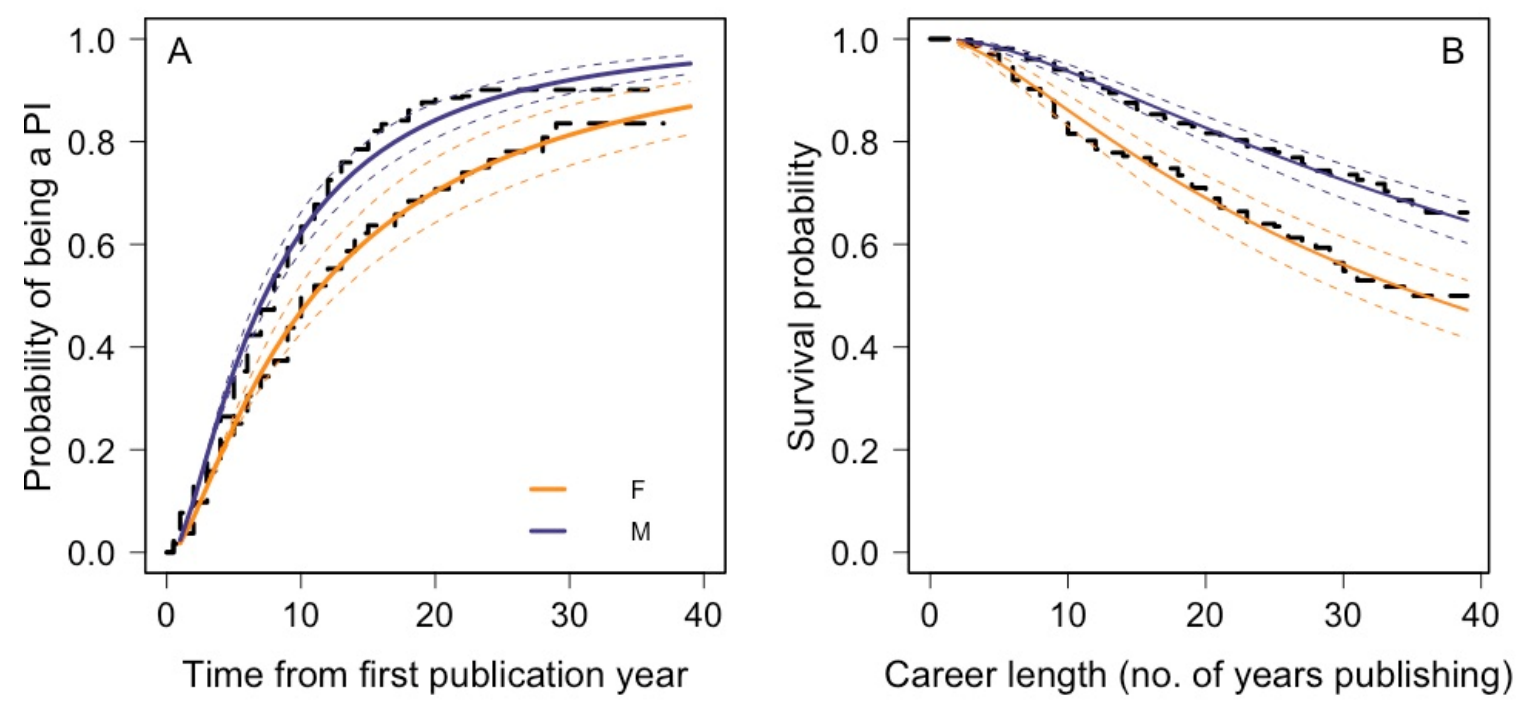

1005

1006 Figure 1 Differences in performance metrics between female $(n=298)$ and male $(n=637)$

1007 focal authors, based on their publishing output. Female focal authors in our dataset were

1008 (A) less likely and took longer to reach PI status, and (B) had shorter scientific careers than

1009 male focal authors, on average (as measured by number of years publishing). Black dashed

1010 lines represent Kaplan-Meier survival functions, while the coloured lines represent model

1011 predictions (solid lines) with 95\% confidence intervals (dotted lines).

1012 


\section{Collaborativeness}

\section{Co-author} connectedness
$\mathbf{F}$
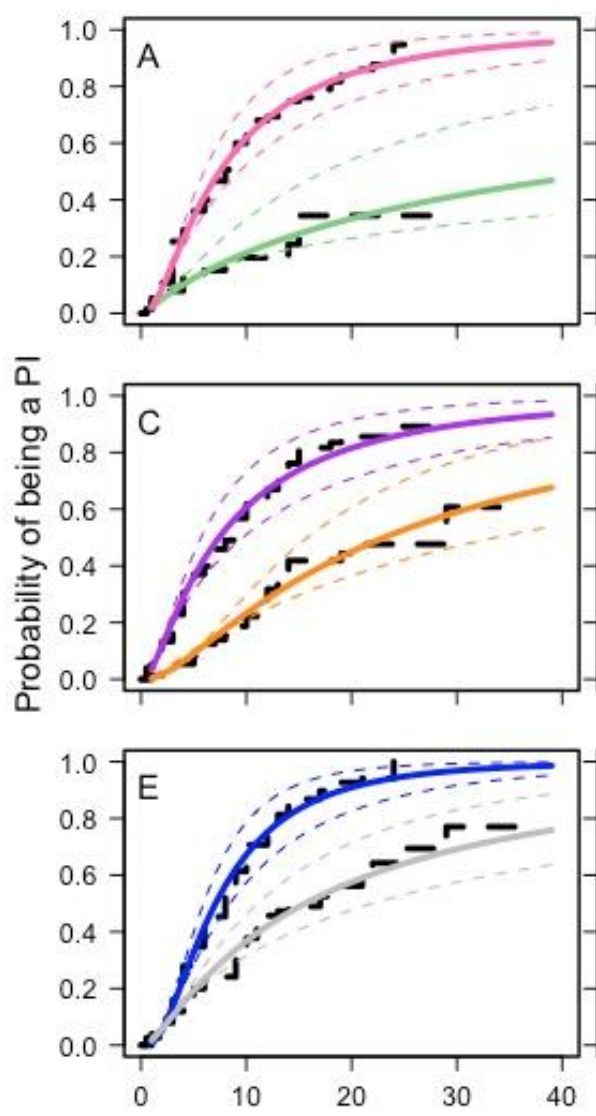

M
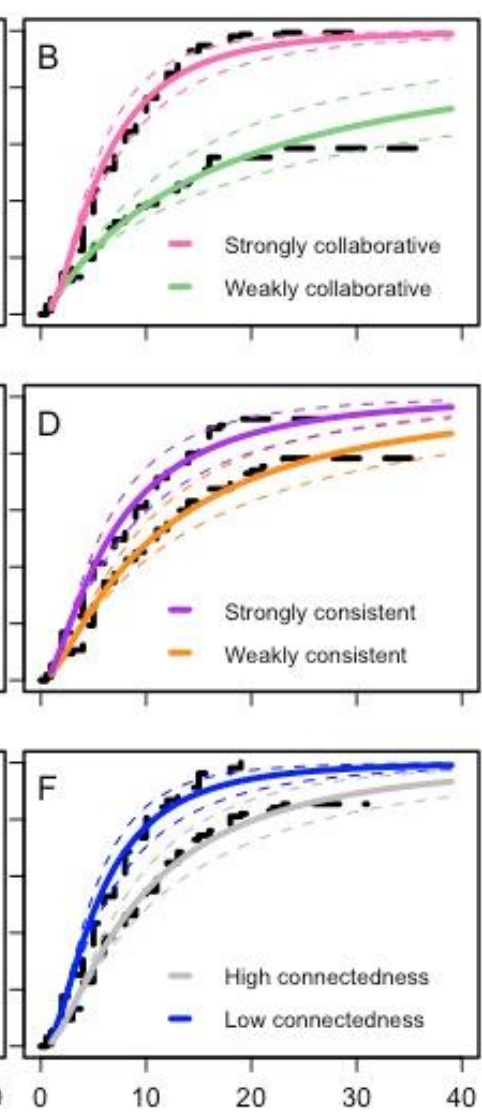

Time from first publication year

1015 Figure 2 The impact of publication output-corrected egocentric network metrics

1016 (collaborativeness $[n=298 \mathrm{~F} ; n=637 \mathrm{M}]$, consistency $[\mathrm{n}=298 \mathrm{~F} ; \mathrm{n}=637 \mathrm{M}]$, co-author

1017 connectedness $[\mathrm{n}=296 \mathrm{~F} ; \mathrm{n}=634 \mathrm{M}]$ ) on the time taken to achieve PI status (expressed as

1018 difference in years between the first publication and the second last-author authored

1019 publication), for female and male focal authors. Analyses were conducted on continuous

1020 data, but the two lines visualised in each plot represent the $25^{\text {th }}$ and $75^{\text {th }}$ quartile of the data

1021 for each variable. Black dashed lines represent Kaplan-Meier survival functions, while the

1022 coloured lines represent model predictions (solid lines) with 95\% confidence intervals

1023 (dotted lines). 


\section{Collaborativeness}

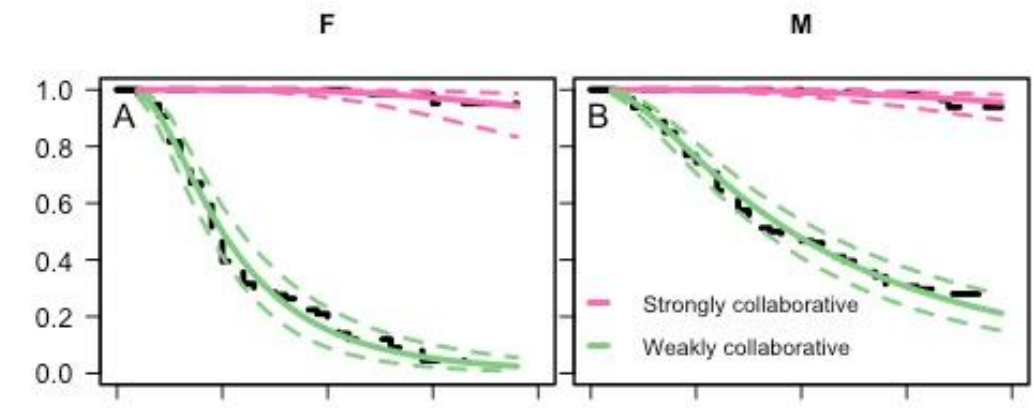

\section{Co-author} connectedness
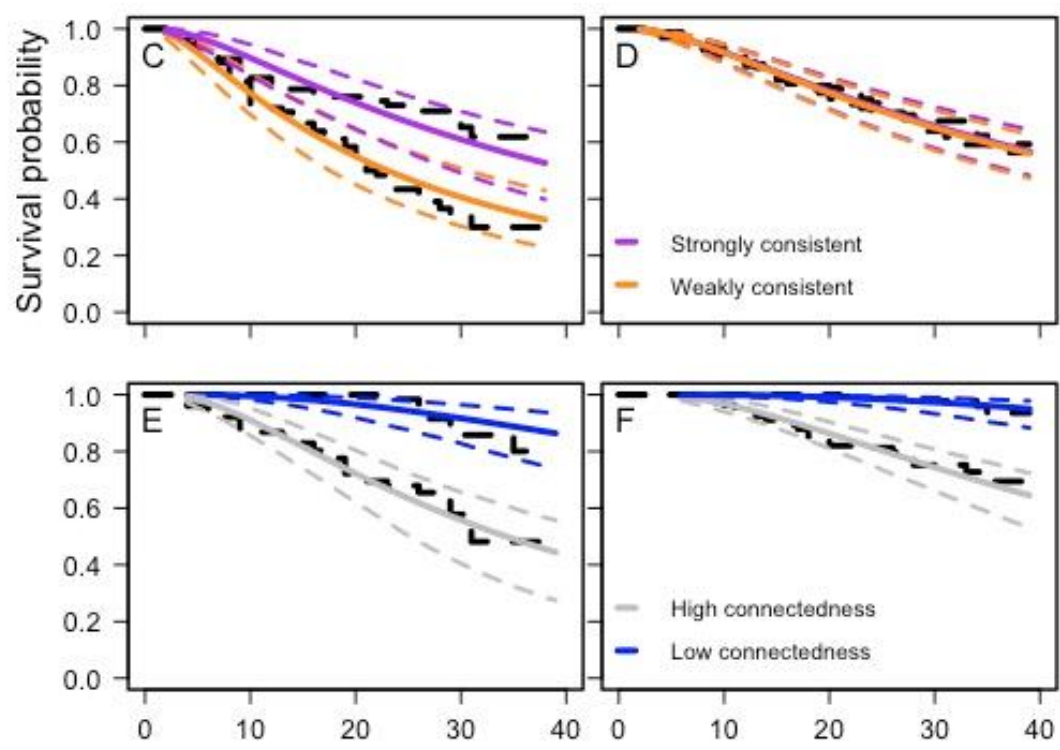

Career length (no. of years publishing)

1026

1027 Figure 3 The impact of publication output-corrected egocentric network metrics

1028 (collaborativeness $[n=298 \mathrm{~F} ; n=637 \mathrm{M}]$, consistency $[\mathrm{n}=298 \mathrm{~F} ; \mathrm{n}=637 \mathrm{M}]$, co-author

1029 connectedness $[n=296 \mathrm{~F} ; n=634 \mathrm{M}]$ ) on career length (expressed as publication years),

1030 for female and male focal authors. Analyses were conducted on continuous data, but the

1031 two lines visualised in each plot represent the $25^{\text {th }}$ and $75^{\text {th }}$ quartile of the data for each

1032 variable. Black dashed lines represent Kaplan-Meier survival functions, while the coloured

1033 lines represent model predictions (solid lines) with 95\% confidence intervals (dotted lines). 


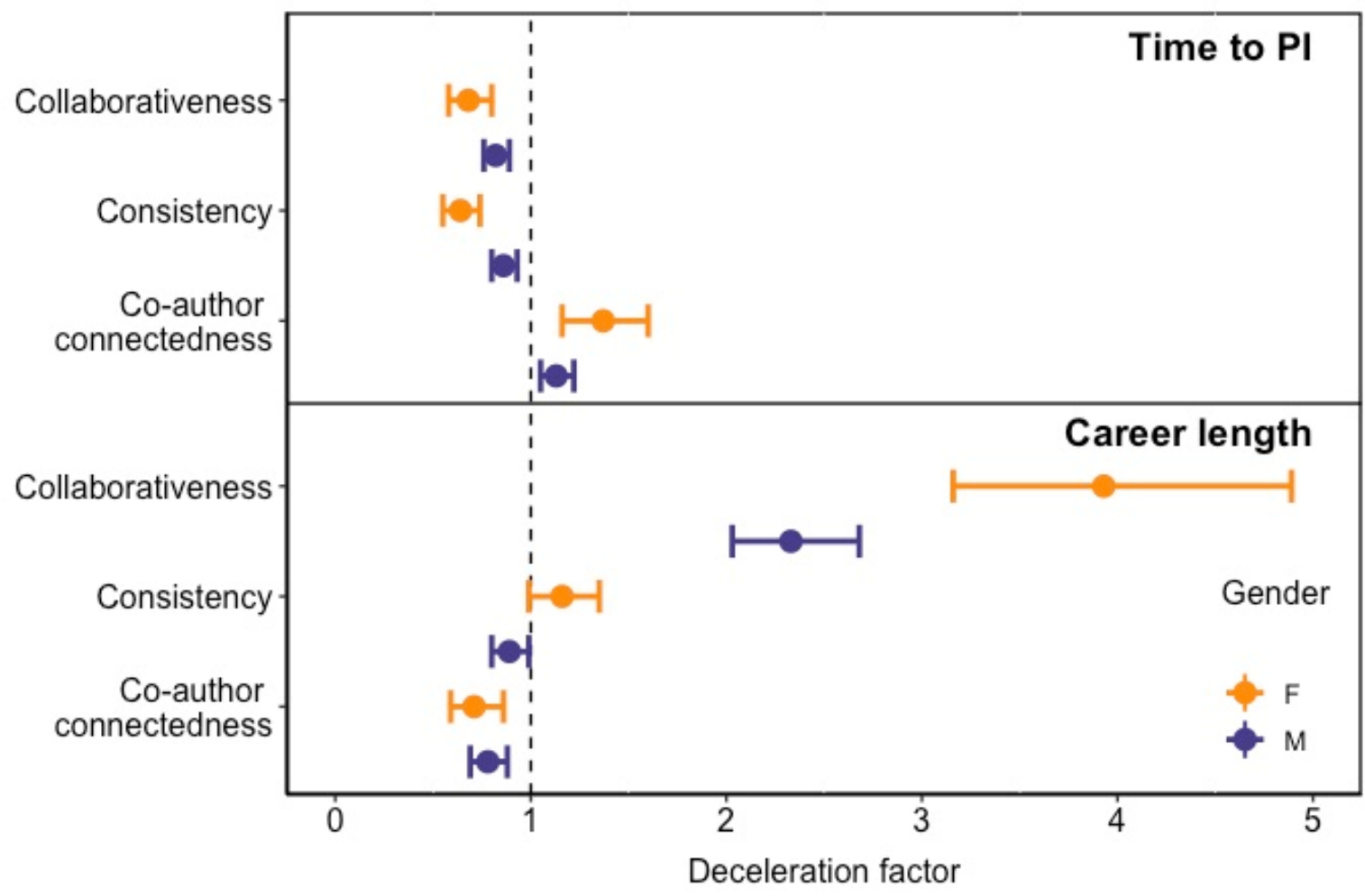

1036

1037 Figure 4 The relative effect size of publication output-corrected egocentric network metrics (collaborativeness $[n=298 \mathrm{~F} ; n=637 \mathrm{M}]$, consistency $[\mathrm{n}=298 \mathrm{~F} ; \mathrm{n}=637 \mathrm{M}]$, coauthor connectedness $[\mathrm{n}=296 \mathrm{~F} ; \mathrm{n}=634 \mathrm{M}])$ on the time taken to achieve PI status, and

1040 career length, for female and male focal authors. The deceleration factor represents the

1041 AFT model estimate (based on separate models for female and male focal authors), and

1042 thus provides an indication of the magnitude and direction of effect of a particular

1043 egocentric network metric on our two measures of scientific performance. Filled symbols

1044 indicate the estimate, with error bars representing 95\% confidence intervals. Estimates to

1045 the left of the dotted vertical line $(<1.0)$ indicate a reduction in the time to the parameter of

1046 interest (i.e. reduced time to PI, or career length), and vice versa for estimates $>1.0$.

1047 Estimates with confidence intervals overlapping 1.0 are non-significant while, within a 1048 particular social metric, overlapping confidence intervals imply that the estimates do not 1049 differ significantly between females and males. 


\section{SUPPLEMENTARY MATERIALS}

1051

\section{Supplementary methods S1}

1053 Our final dataset included 298 women (32\%) and 637 men (68\%), and three focal authors

1054 for whom gender was unknown. Focal authors came from 39 different countries, with the majority from Europe (47\%) or North America (35\%). The mean \pm SE number of unique co-authors per focal author was $81.09 \pm 2.72$ (median $=58$; range $=1-628$; Fig. S3), with each focal author producing, on average, $57.03 \pm 1.85$ papers (median $=42$; range $=3$ 375 papers; Fig. S3). This equates to $3.86 \pm 0.04$ co-authors per paper (median $=3.72$ co-

1059 authors; range $=1.11-11.11)$. Focal authors published with the same co-author(s) an average of $2.02 \pm 0.02$ times $($ median $=1.90$; range $=1-7$; Fig. S3). It took, on average, $9.34 \pm 0.22$ years for a focal author to become a PI, ranging from less than one year to 37 years (median $=8$ years). The mean career lifespan was $24.67 \pm 0.29$ years $($ median $=26$ years; range $=2-39$ ).

1064

\section{Supplementary methods S2}

1066 Due to the Scopus database and the Scopus Database 'API' Interface not being completely

1067 synchronised, we had to manually download publication details for 292 authors (24\% of

1068 total) directly from the Scopus website, using the 'search by Author ID' function. To be

1069 sure that these two methods yielded identical results that could be merged, we extracted

1070 publication details using both methods for a random selection of 120 Scopus Author

1071 Identifiers. We found only two authors $(<2 \%)$ that differed in the number of papers

1072 assigned to them by the two search methods (one additional, and one fewer paper,

1073 respectively). We consider this validates combining data extracted via these two different

1074 methods. 


\section{Supplementary methods S3}

1076 We applied a series of restrictions to our dataset of focal authors (see Methods for details),

1077 and for some survival models, we censored data. We checked to ensure that these

1078 restrictions and censoring decisions did not result in a gender bias through one gender

1079 being disproportionately excluded from the final dataset or particular analyses. The final

1080 dataset consisted of $32 \%$ women $(n=298)$. For every restriction in place (see below for a

1081 breakdown of numbers), we therefore confirmed that the percentage of women excluded or

1082 affected was not greater than $32 \%$.

1083

1084 Checking for gender bias in restricted data:

1085

1086

1087

1088

1089

1090

1091

1092

1093

1094

1095

1096

1097

1098

- Focal authors that only published single-author papers:

$$
\mathrm{n}=9 \text { authors excluded; } 78 \% \text { women }(7 \text { women and } 2 \text { men)* }
$$

- Focal authors that published fewer than three papers in total:

$\mathrm{n}=91$ authors excluded; $55 \%$ women (50 women, 40 men, 1 unknown gender)*

- Focal authors with more than 400 papers:

$\mathrm{n}=6$ authors excluded; $17 \%$ women (one woman and five men)

- Focal authors that published their first paper before 1980:

$$
\mathrm{n}=192 \text { authors excluded; } 11 \% \text { women (22 women and } 170 \text { men) }
$$

- Non-journal articles:

0 authors excluded but 394 authors affected; 22\% women (85 women and 309 men). For the women affected, $5.01 \% \pm 0.66 \%($ mean \pm SE) of their total paper output were excluded, while for men this was $3.86 \pm 0.20 \%$ of their papers (mean $\pm \mathrm{SE}$ ).

- Articles with more than 20 or more authors:

0 authors excluded but 330 authors affected. 
$1099 *$ Although these restrictions are biased towards exclusion of female focal authors, they are

1100 both methodologically necessary in order to enable the construction of meaningful

1101 egocentric networks.

1102

1103 Checking for gender bias in censored data for survival analyses:

1104 We applied three censoring cut-offs to our data, when determining the survival times of

1105 focal authors in our dataset: still publishing in 2012, in 2014 or in 2016. Using a more

1106 conservative censoring date did not change the proportion of female focal authors that

1107 were considered to have left science.

1108 - 2012: 223 authors censored, 42.2\% women (94 women, 127 men, 2 unknown gender)

1109 - 2014: 252 authors censored, 42.1\% women (106 women, 144 men, 2 unknown gender)

1110 - 2016: 299 authors censored; 42.5\% women (127 women, 169 men, 3 unknown gender) 

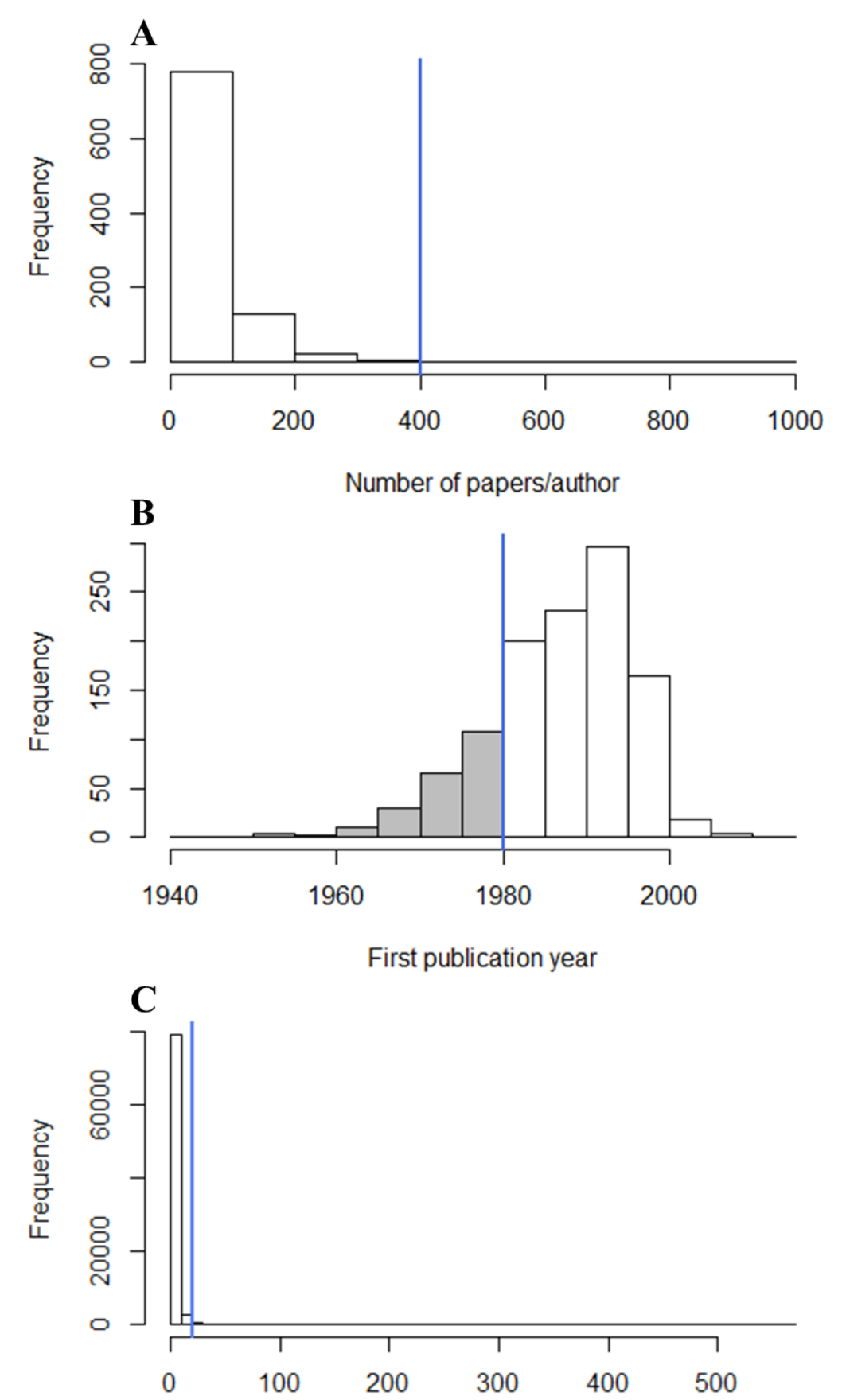

1112

Number of authors/paper

1113 Figure S1 Visualisation of the restrictions that were applied to the final dataset. Blue lines

1114 indicate the implemented cut-off points and shaded areas indicate excluded data. (A)

1115 Papers with $\leq 400$ authors were excluded. (B) Authors that started publishing on or before

11161980 were excluded. (C) Papers with $\leq 20$ co-authors were excluded. 


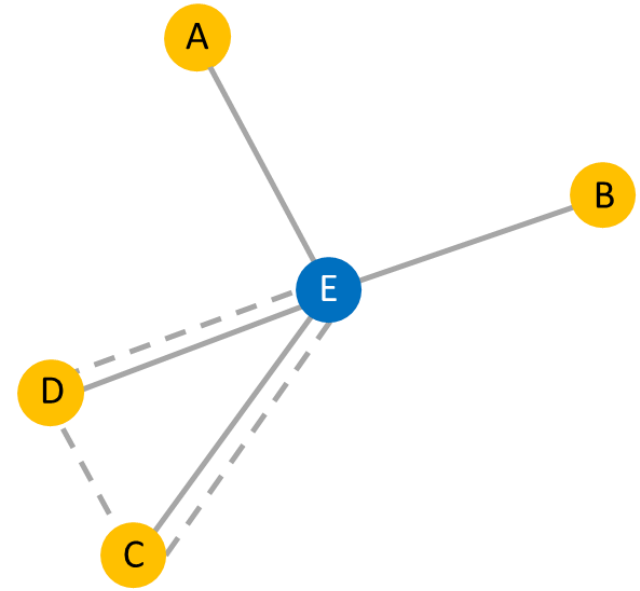

1118 Figure S2 An anonymised egocentric network from our dataset with connections between

1119 the ego (focal author; blue dot, E) and co-authors (yellow dots, A-D) represented by grey

1120 lines. In this example, the focal author has published 5 papers in total: a single paper with

1121 each co-author, and a joint paper with co-authors C and D (represented by the dotted line

1122 joining E, C and D). Thus, focal author E has a network size or 'degree' of 5 (5 unique co-

1123 authors), and a tie strength of 1.25 (E has published, on average, $5 / 4=1.25$ times per co-

1124 author). The global clustering coefficient for focal author $\mathrm{E}=3 \mathrm{x}$ number of triangles (one

1125 triangle: E-D-C)/number of all triplets (closed; E-D-C; D-E-C; D-C-E and open: A-E-B; C-

1126 E-B; D-E-B; C-E-A; D-E-A $)=(3 \times 1) / 8=0.375$. After correcting for publication output

1127 (see 'Accounting for the effect of publication number on social metrics' in the Methods

1128 section) focal author $\mathrm{E}$ has a collaborativeness score of -1.27 (range: -6.62, 4.13), a

1129 consistency score of -1.28 (range: $-2.61,5.32$ ), and a connectedness score of - 0.08 (range -

$11305.42,4.43)$. These are all negative values, implying that, compared to the average for other

1131 focal authors with the same number of publications, $\mathrm{E}$ is less collaborative, less consistent

1132 in who they publish with, and has co-authors that are relatively unconnected. Such a

1133 combination of social metric scores would suggest that focal author E probably did not

1134 become a PI, or else took a relatively long time to progress to this career stage, and may

1135 not have survived for particularly long in their career. 

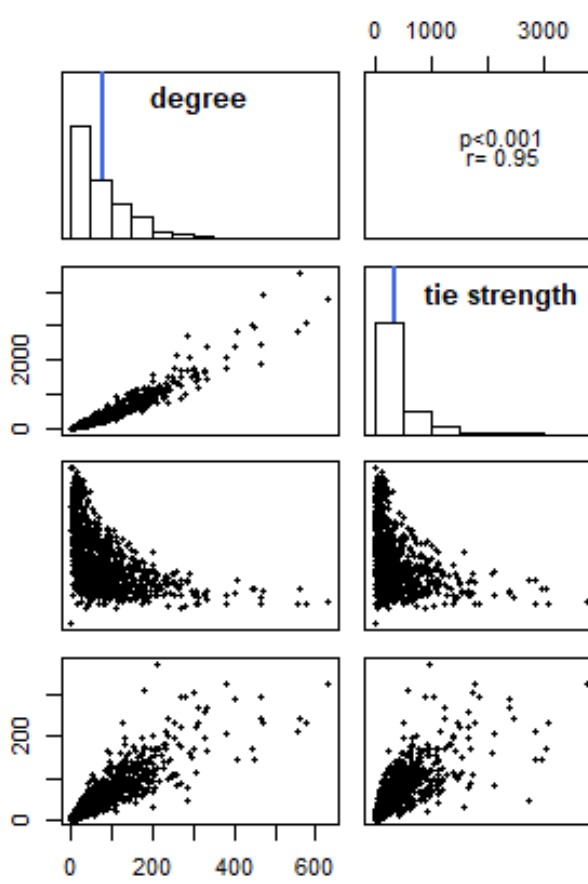
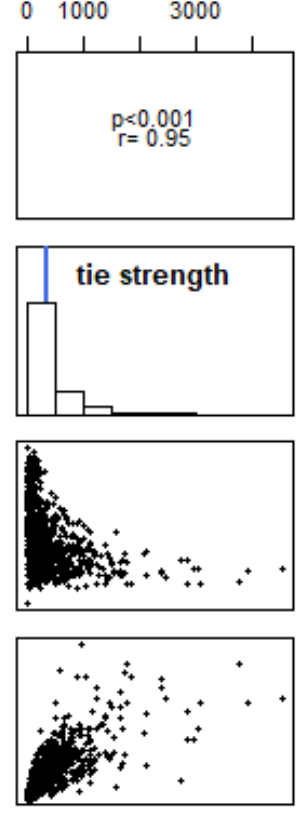
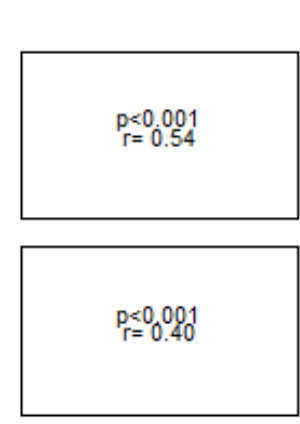

gl. clustering coeff.
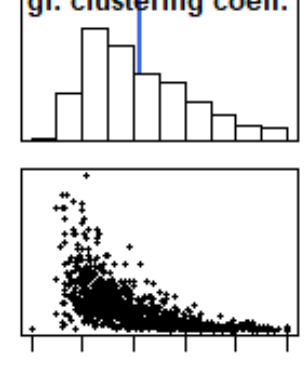

$\begin{array}{llllll}0.0 & 0.2 & 0.4 & 0.6 & 0.8 & 1.0\end{array}$
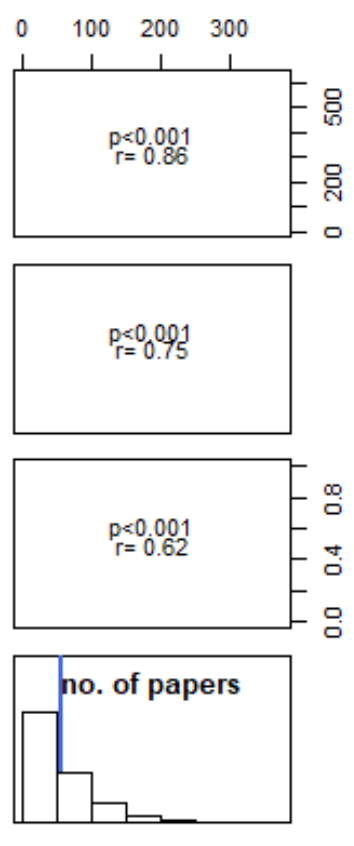

1137 Figure S3 Correlation matrix and frequency histograms of the social network metrics

1138 measured, and number of papers $(n=938$ focal authors $)$. Blue lines in graphs indicate the

1139 mean value for each variable.

1140 


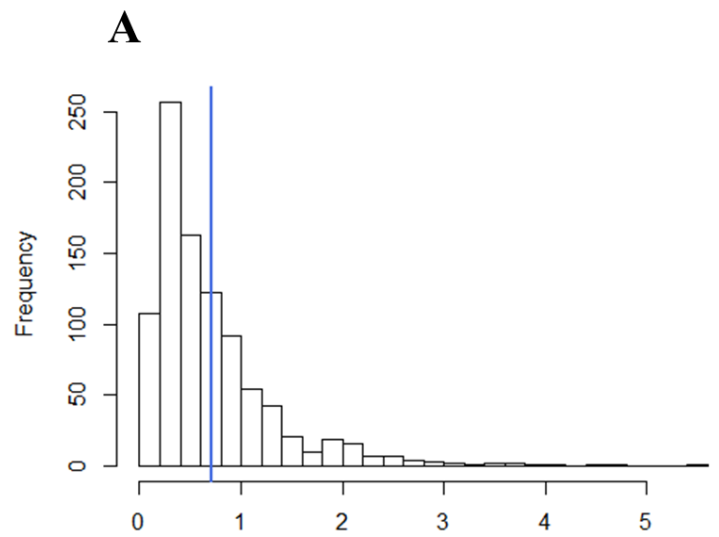

$\%$ of other ego networks focal authors are included in
B

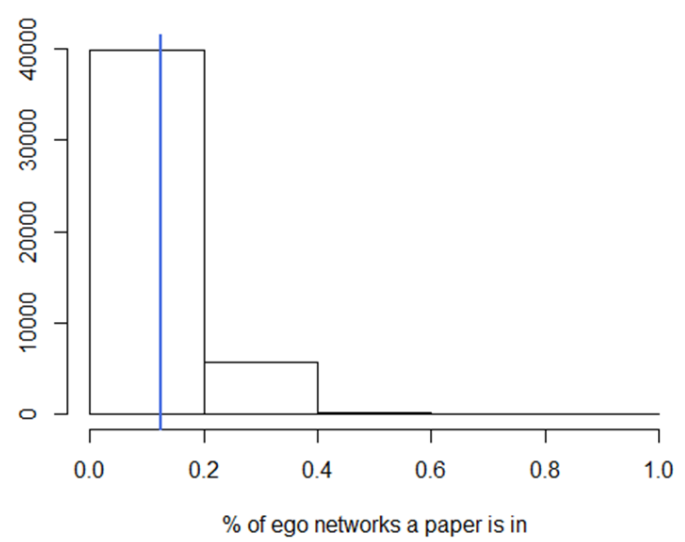

1142 Figure S4 (A) A frequency histogram of the percentage of other ego networks that focal

1143 authors are included in. (B) A frequency histogram of the percentage of ego networks that

1144 individual papers appear in. In both panels the blue line represents the mean.

1145 

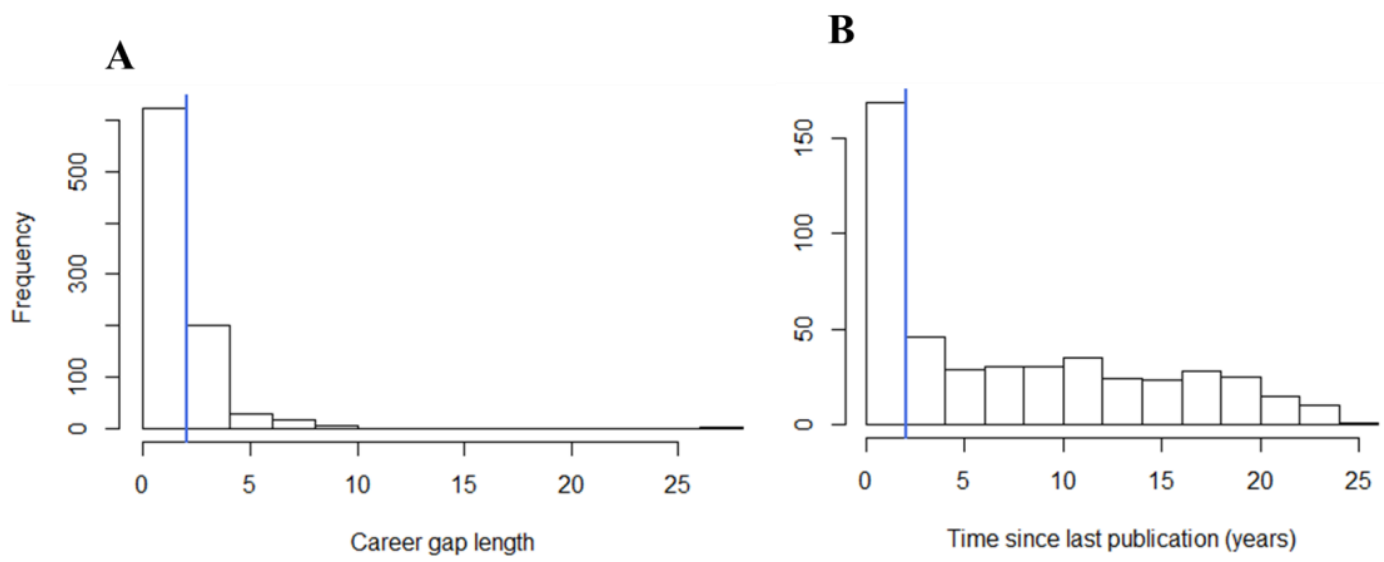

1147 Figure S5 Censoring in the survival models. We censored focal authors $(n=938)$ that

1148 were still publishing in $\geq 2016$, given that two years is the most common length of a career

1149 gap in the dataset, and the clear drop off in number of authors that published more than 2

1150 years ago. We assume that all authors that published for the last time before 2016 were no

1151 longer actively contributing to science, and from the perspective of our survival analyses

1152 are thus 'dead'. (A) Length of all career gaps (i.e. years between publications); (B) Years

1153 since last publication. In both panels the blue line indicates the two-year cut-off. 

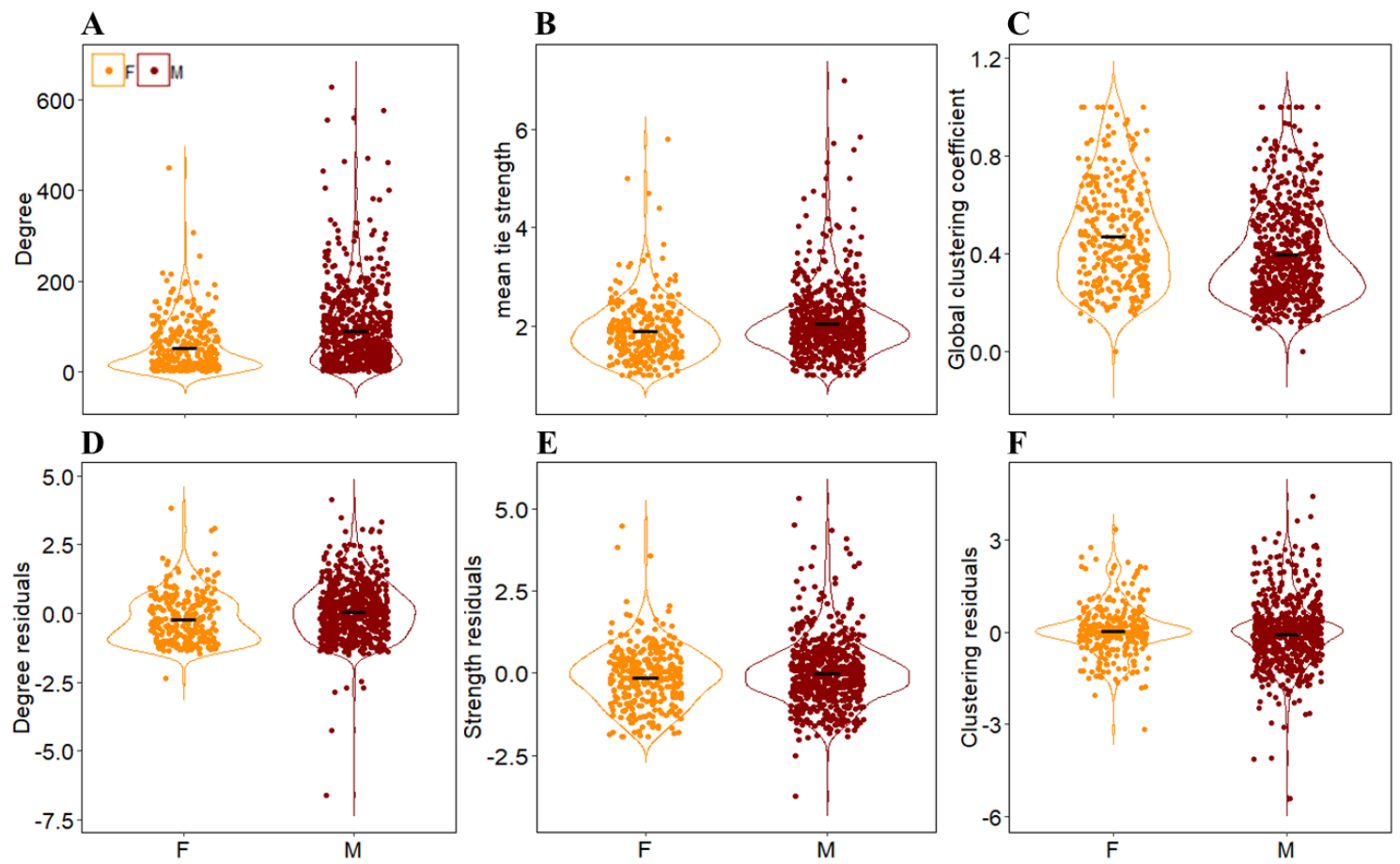

1156 Figure S6 Significant gender differences in our social network metrics exist before

1157 correcting for number of papers (A-C). After correcting for publication output, males ( $\mathrm{n}=$

1158 637) were found to be significantly more collaborative than females $(n=298)(D)$, while

1159 gender did not significantly affect consistency and co-author connectedness scores (E-F).

1160 Violin plots visualise the distribution of the data and its probability density. All raw

1161 (scaled) data points are displayed, and the black line indicates the mean.

1162

1163 
bioRxiv preprint doi: https://doi org/10.1101/2020.03.02 973479; this version posted March 4,2020 . The copyright holder for this preprint (which was not certified by peer review) is the author/funder, who has granted bioRxiv a license to display the preprint in perpetuity. It is made available under aCC-BY-NC-ND 4.0 International license.

1164 Table S1 Output from AFT models testing the relationship between career length and each

1165 of the three measures of co-authorship sociality, in which we assumed authors to have

1166 stopped publishing when their last publication was before 2012, before 2014 or before

1167 2016. Shaded estimates indicate non-significant relationships (95\% confidence intervals

1168 overlapping 1.0).

1169

Estimate $[95 \% \mathrm{CI}]$

\begin{tabular}{ccccc} 
& Term & $\mathbf{2 0 1 2}$ & $\mathbf{2 0 1 4}$ & $\mathbf{2 0 1 6}$ \\
\hline \multirow{2}{*}{ Collaborativeness } & Female & $7.70[5.25,11.29]$ & $5.83[4.30,7.92]$ & $4.00[3.23,4.96]$ \\
& Male & $2.91[2.38,3.56]$ & $2.71[2.27,3.23]$ & $2.33[2.02,2.68]$ \\
\hline \multirow{2}{*}{ Consistency } & Female & $1.14[0.93,1.40]$ & $1.12[0.37,1.35]$ & $1.13[0.97,1.32]$ \\
& Male & $0.86[0.76,0.97]$ & $0.86[0.76,0.97]$ & $0.89[0.80,0.98]$ \\
\hline \multirow{2}{*}{ Co-author } & Interaction & $1.06[0.78,1.43]$ & $1.03[0.78,1.35]$ & $1.13[0.90,1.41]$ \\
& Female & $0.72[0.56,0.95]$ & $0.74[0.59,0.94]$ & $0.70[0.58,0.85]$ \\
connectedness & Male & $0.77[0.650 .90]$ & $0.76[0.66,0.88]$ & $0.78[0.69,0.88]$
\end{tabular}

1170

1171

1172 
1173 Table S2 Output from AFT models testing how career length is influenced by the three

1174 measures of co-authorship sociality, as calculated over the first ten years for authors that

1175 started publishing just before or after their first ISBE conference attendance. Shaded

1176 estimates indicate non-significant relationships (95\% confidence intervals overlapping

1177 1.0).

1178

\begin{tabular}{lll}
\hline & \multicolumn{1}{c}{ Term } & Estimate $[\mathbf{9 5 \%}$ CI $]$ \\
\hline Collaborativeness & Fnteraction & $0.92[0.68,1.23]$ \\
& Male & $1.37[1.10,1.71]$ \\
& Interaction & $0.78[0.59,1.02]$ \\
Consistency & Female & $0.95[0.76,1.18]$ \\
& Male & $0.73[0.62,0.87]$ \\
\hline Co-author connectedness & Female & $0.82[0.69,0.98]$ \\
& Male & $0.75[0.61,0.93]$ \\
& & \\
\hline
\end{tabular}

1179 